\title{
Hydrodynamic fluctuation-induced forces in confined fluids
}

\author{
Christopher Monahan, ${ }^{1}$ Ali Naji, ${ }^{2}$ Ronald Horgan, ${ }^{3}$ Bing-Sui Lu, ${ }^{4}$ and Rudolf Podgornik ${ }^{4,5,6}$ \\ ${ }^{1}$ Department of Physics and Astronomy, University of Utah, Salt Lake City, Utah 84112, USA \\ ${ }^{2}$ School of Physics, Institute for Research in Fundamental Sciences (IPM), Tehran 19395-5531, Iran \\ ${ }^{3}$ DAMTP, Centre for Mathematical Sciences, University of Cambridge, Cambridge, CB3 0WA, United Kingdom \\ ${ }^{4}$ Department of Theoretical Physics, J. Stefan Institute, SI-1000 Ljubljana, Slovenia \\ ${ }^{5}$ Department of Physics, Faculty of Mathematics and Physics, \\ University of Ljubljana, SI-1000 Ljubljana, Slovenia \\ ${ }^{6}$ Department of Physics, University of Massachusetts, Amherst, MA 01003, USA
}

\begin{abstract}
We study thermal, fluctuation-induced hydrodynamic interaction forces in a classical, compressible, viscous fluid confined between two rigid, planar walls with no-slip boundary conditions. We calculate hydrodynamic fluctuations using the linearized, stochastic Navier-Stokes formalism of Landau and Lifshitz. The mean fluctuation-induced force acting on the fluid boundaries vanishes in this system, so we evaluate the two-point, time-dependent force correlations. The equal-time correlation function of the forces acting on a single wall gives the force variance, which we show to be finite and independent of the plate separation at large inter-plate distances. The equal-time, cross-plate force correlation, on the other hand, decays with the inverse inter-plate distance and is independent of the fluid viscosity at large distances; it turns out to be negative over the whole range of plate separations, indicating that the two bounding plates are subjected to counter-phase correlations. We show that the time-dependent force correlations exhibit damped temporal oscillations for small plate separations and a more irregular oscillatory behavior at large separations. The long-range hydrodynamic correlations reported here represent a "secondary Casimir effect", because the mean fluctuation-induced force, which represents the primary Casimir effect, is absent.
\end{abstract}

PACS numbers: 47.35.-i,05.40.-a,05.20.Jj

\section{INTRODUCTION}

The Casimir effect [1] is the most important example of a slew of phenomena usually referred to as fluctuationinduced interactions, their phenomenology extending from cosmology on the one side to nanoscience on the other 2 29]. The general idea tying these diverse phenomena together is that the confining surfaces constrain the quantum and thermal field fluctuations, inducing longrange interactions between these boundaries [3, 4] . For electromagnetic fields, these confinement effects lead to the Casimir-van der Waals interactions that can be derived within the specific framework of QED, and quantum field theory more generally [6]. Inspired by the close analogy between thermal fluctuations in fluids and quantum fluctuations in electromagnetism, Fisher and de Gennes predicted the existence of long-range fluctuation forces in other types of critical condensed matter systems [10] and the terms "Casimir" or "Casimir-like effect" now denote a range of other non-electromagnetic fluctuationinduced forces $[3,9]$.

Beyond detailed measurements of the Casimir-van der Waals interactions [6], attention has been directed toward Casimir-like forces engendered by density fluctuations in the vicinity of the vapor-liquid critical point [8, 11, 12]; in binary liquid mixtures near the critical demixing point

\footnotetext{
* Current address: New High Energy Theory Center, Rutgers, The State University of New Jersey, 136 Frelinghuysen Road, Piscataway, NJ 08854-8019
}

13, 14]; and in thin polymer 15-17] and liquid crystalline films [18, 19]. Most recently, several studies have examined fluctuation-induced interactions for the CasimirLifshitz force out of thermal equilibrium [20, 21], for the temporal relaxation of the thermal Casimir or van der Waals force 22], and for nonequilibrium steady states in fluids [23 25], where fluctuations are anomalously large and long-range.

It is instructive to recall that the original 1955 derivation of the electromagnetic Casimir-van der Waals interactions by Lifshitz [26] was not fundamentally rooted in QED but rather in stochastic electrodynamics, first formulated by Rytov [27]. In stochastic electrodynamics, Maxwell's equations are augmented by fluctuating displacement current sources 28]. This leads to two coupled electrodynamic Langevin-type equations, for each of the fundamental electrodynamic fields, that are then solved with standard boundary conditions. The interaction force is obtained by averaging the Maxwell stress tensor and taking into account the statistical properties of the fluctuating sources [29]. This paradigmatic Lifshitzroute to fluctuation-induced interactions later became disfavored as other formal approaches gained strength [6], but appears to be reborn in recent endeavors regarding non-equilibrium fluctuation-induced interactions 23, 24]. In fact, in the Dean-Gopinathan method there exists a mapping of the non-equilibrium problem characterized by dissipative dynamics onto a corresponding static (Lifshitz) partition function provided by the Laplace transform of the time-dependent force and the static partition function [30, 31].

Based on the success of stochastic electrodynamics, 
Landau and Lifshitz proposed by analogy the stochastic dissipative hydrodynamic equations [32], augmenting the linearized Navier-Stokes equations with fluctuating heat flow vector and fluctuating stress tensor [25, 33]. This leads to three coupled hydrodynamic equations involving the fundamental hydrodynamic fields of mass density, velocity and local temperature, which can now be solved in different contexts. In the absence of thermal conductivity, this system further reduces to a Langevin-type equation for the velocity field, involving the stress tensor fluctuations, and a continuity equation for the mass density field. Since the fundamental hydrodynamic equations are non-linear, the derivation of fluctuating Landau-Lifshitz hydrodynamics already involves heavy linearity Ansätze and the possible generalization to a full non-linear fluctuating hydrodynamics is not clear [34, 35].

Although fluctuating electrodynamics is based on linear Maxwell's equations with stresses quadratic in the field and fluctuating hydrodynamics stems from nonlinear Navier-Stokes equations with stresses linear in velocities, the general similarity between these approaches might nevertheless lead one to assume that, in confined geometries, there should exist Casimir-like hydrodynamic fluctuation forces. But this notion is at odds with the standard decomposition of the classical partition function into momentum and configurational parts. This decomposition has far-reaching consequences, which were clearly understood as far back as van der Waals' thesis [36]. While there is an analogy between the description of fluctuations in these two areas of physics, caution should be exercised when trying to translate results from one field directly into the other. We will show that there does exist a type of Casimir effect in the hydrodynamic context, but that this effect has fundamentally different properties from the conventional Casimir effect.

The first step in bringing together the Casimir force in electrodynamics and its putative counterpart in hydrodynamics was made by Jones [37]. Inspired by the obvious analogy between electrodynamics and hydrodynamics, Jones investigated the possible existence of a longranged, fluctuation-induced, effective force generated by confining boundaries in a fluid. He showed that in linearized hydrodynamics the net (mean) stochastic force vanishes, which led him to introduce a next-to-leading order formalism. The status of this formalism, however, is not entirely clear, because there are linearity assumptions rooted deep within fluctuational hydrodynamics [25, 33]. Within the context of this next-to-leading order formalism, Jones demonstrated that long-range forces could exist in a semi-infinite fluid or around an immersed spherical body, and would be strongest in incompressible fluids, with much weaker forces in compressible fluids. This result is at odds with the momentum decomposition of the classical partition function and should be considered an artifact of the next-to-leading order analysis of the stochastic equations governing the hydrodynamic field evolution.

Chan and White [38], therefore, reconsidered the whole calculation. They concentrated on the planar geometry of two hard walls immersed in a fluid and argued that hydrodynamic fluctuations could give rise to a repulsive force in incompressible fluids, but that this force would vanish for classical compressible fluids. Since an incompressibility Ansatz does not translate directly into the interaction potential in the classical partition function [39], this fictional case could lead to a fluctuationinduced interaction that would not be contrary to the argument based on the momentum decomposition of the classical partition function. The repulsive fluctuationinduced force would also in itself not be that hard to envision since the existence of a repulsive force in the context of van der Waals interactions is well-established and was originally proposed in Ref. [40]. The vanishing of the fluctuation-induced force for classical compressible fluids is based on a rough argument of analytic continuation of the viscosities into the infinite frequency domain [38]. While this latter argument is appropriate in electrodynamics, because an infinite frequency corresponds to the vacuum, it is not reasonable in hydrodynamics, where the whole basis of the continuum hydrodynamic theory breaks down before any such limit could be enforced [33].

Therefore, both approaches to the problem of hydrodynamic Casimir-like interactions have strong limitations and subsequent developments failed to conclusively prove either point of view [41].

In this paper, we revisit the question of the existence of long-range, fluctuation-induced forces in classical fluids. We work strictly within the framework of linearized stochastic hydrodynamics and rather than considering the net force, which is zero trivially, we study the force correlators. In other words, we focus on the question: In what way do boundary conditions and statistical properties of the fluctuating hydrodynamic stresses affect the statistical properties (correlators) of the random forces acting on the bounding surfaces?

We formulate a general approach to this problem by considering a fluid of arbitrary compressibility, bounded between two plane-parallel, hard walls with no-slip boundary conditions. Thermal fluctuations lead to spatio-temporal variations in the pressure and velocity fields that can be calculated using the linearized, stochastic Navier-Stokes formalism of Landau and Lifshitz [32]. Within this approach, we derive analytical expressions for the time-dependent correlators (for both the sameplate and the cross-plate correlators) of the fluctuationinduced forces acting on the walls. In particular, we express the variance of these forces in terms of frequency integrals that have simple plate-separation dependence in the small and large plate-separation limits.

Our results do not depend upon the next-to-leading order formalism of Jones [37], nor do they depend on the unrealistic validity of analytic continuation of the viscosities in the whole frequency domain [38]. We show that, while the mean force vanishes, the variance of the fluctuation-induced normal force is finite and depends on the separation between the bounding surfaces. We 
call this the secondary Casimir effect, because the primary Casimir effect refers to the average value of the fluctuation-induced force (which is zero here) and not strictly its variance. Both quantities have been investigated in other Casimir-like situations 22, 42] and in disordered charged systems [43 45]. The equal-time, crossplate force correlation exhibits long-range behavior that is independent of the fluid viscosity and decays proportional to the inverse plate separation. Finally, we find that the time-dependent correlators exhibit damped oscillatory behavior for small plate separations that becomes irregular at large distances.

In Sec. III, we outline the stochastic formalism of Landau and Lifshitz and the strategy of our calculation of hydrodynamic fluctuation-induced forces in the general case of compressible fluids. Sections [II and IV present the main steps of our calculation. We show results for the equal-time force correlators and the two-point, timedependent correlators in Sections $\mathrm{V}$ and VI, respectively. We conclude our discussions in Sec. VII

\section{FORMALISM}

We consider the hydrodynamic fluctuations in a Newtonian fluid at rest and in the absence of heat transfer. These fluctuations are described by the stochastic Landau-Lifshitz equations 32]

$$
\begin{aligned}
\eta \nabla^{2} \mathbf{v}+\left(\frac{\eta}{3}+\zeta\right) & \nabla(\nabla \cdot \mathbf{v})-\nabla p \\
& -\rho\left(\frac{\partial \mathbf{v}}{\partial t}+\mathbf{v} \cdot \nabla \mathbf{v}\right)=-\nabla \cdot \mathbf{S} \\
\frac{\partial \rho}{\partial t}+\nabla \cdot(\rho \mathbf{v}) & =0
\end{aligned}
$$

where $\mathbf{v}=\mathbf{v}(\mathbf{r} ; t), p=p(\mathbf{r} ; t)$ and $\rho=\rho(\mathbf{r} ; t)$ are the velocity, pressure and density fields and $\eta$ and $\zeta$ are the shear and bulk viscosity coefficients, respectively [46]. The randomly fluctuating microscopic degrees of freedom are driven by the random stress tensor $\mathbf{S}=\mathbf{S}(\mathbf{r} ; t)$, which is assumed to have a Gaussian distribution with zero mean $\left\langle S_{i j}(\mathbf{r} ; t)\right\rangle=0$ and the two-point correlator

$$
\begin{aligned}
& \left\langle S_{k l}(\mathbf{r} ; t) S_{m n}\left(\mathbf{r}^{\prime} ; t^{\prime}\right)\right\rangle=2 k_{\mathrm{B}} T \delta\left(\mathbf{r}-\mathbf{r}^{\prime}\right) \delta\left(t-t^{\prime}\right) \\
& \quad \times\left[\eta\left(\delta_{k m} \delta_{l n}+\delta_{k n} \delta_{l m}\right)-\left(\frac{2 \eta}{3}-\zeta\right) \delta_{k l} \delta_{m n}\right] .
\end{aligned}
$$

Here the subindices $(i, j, k, \ldots)$ denote the Cartesian components $(x, y, z), k_{\mathrm{B}}$ is Boltzmann's constant and $\langle\cdots\rangle$ denote an equilibrium ensemble average at temperature $T$. We do not consider any possible relaxation effects, which would formally correspond to frequency-dependent viscosities, but these effects can be easily incorporated [32]. Denoting the frequency Fourier transform by a tilde, i.e.,

$$
\widetilde{f}(\omega)=\int \mathrm{d} t e^{i \omega t} f(t)
$$

we have $\left\langle\widetilde{S}_{i j}(\mathbf{r} ; \omega)\right\rangle=0$ and

$$
\begin{gathered}
\left\langle\widetilde{S}_{k l}(\mathbf{r} ; \omega) \widetilde{S}_{m n}^{*}\left(\mathbf{r}^{\prime} ; \omega^{\prime}\right)\right\rangle=4 \pi k_{\mathrm{B}} T \delta\left(\mathbf{r}-\mathbf{r}^{\prime}\right) \delta\left(\omega-\omega^{\prime}\right) \\
\times\left[\eta\left(\delta_{k m} \delta_{l n}+\delta_{k n} \delta_{l m}\right)-\left(\frac{2 \eta}{3}-\zeta\right) \delta_{k l} \delta_{m n}\right]
\end{gathered}
$$

which hold independent of the boundary conditions imposed on the fluid system.

Before proceeding further, we should note that this form of fluctuating hydrodynamics is analogous to the Rytov fluctuating electrodynamics [26], where the basic equations for the electric and magnetic fields are

$$
\begin{aligned}
& \nabla \times \mathbf{E}(\mathbf{r}, t)=-\frac{\partial \mathbf{B}(\mathbf{r}, t)}{\partial t}, \\
& \nabla \times \mathbf{H}(\mathbf{r}, t)=\frac{\partial \mathbf{D}(\mathbf{r}, t)}{\partial t}+\frac{\partial \mathbf{K}(\mathbf{r}, t)}{\partial t},
\end{aligned}
$$

supplemented by $\nabla \cdot \mathbf{D}(\mathbf{r}, t)=0$ and $\nabla \cdot \mathbf{B}(\mathbf{r}, t)=0$ and appropriate boundary conditions. In this case, the fluctuating random polarization, $\mathbf{K}(\mathbf{r} ; t)$, has Gaussian properties with $\left\langle\widetilde{K}_{i}(\mathbf{r} ; \omega)\right\rangle=0$, and

$$
\left\langle\widetilde{K}_{i}(\mathbf{r} ; \omega) \widetilde{K}_{j}^{*}\left(\mathbf{r}^{\prime} ; \omega^{\prime}\right)\right\rangle=k_{\mathrm{B}} T \frac{\varepsilon_{\mathrm{I}}(\omega)}{\omega} \delta_{i j} \delta\left(\mathbf{r}-\mathbf{r}^{\prime}\right) \delta\left(\omega-\omega^{\prime}\right),
$$

where we have assumed a dispersive dielectric response function $\varepsilon(\omega)=\varepsilon_{\mathrm{R}}(\omega)+i \varepsilon_{\mathrm{I}}(\omega)$. We can immediately see the similarity between Eqs. (10)-(5) and Eqs. (6)-(8).

Thus, the stochastic approach to hydrodynamics is very close to Lifshitz's original analysis of the electromagnetic problem [26], provided one fully takes into account the basic differences between the Maxwell equations and the Navier-Stokes equations [38]: The former are linear in the fields with stresses quadratic in the fields, while the latter are non-linear in the fields with stresses linear in the fields. This difference leads to some important distinctions and precludes directly applying results from electrodynamics to the hydrodynamic domain.

\section{A. Linearized stochastic hydrodynamics}

For vanishing random stress tensor, the equilibrium solution of Eqs. (11) and (2) is $\mathbf{v}=\mathbf{0}, p=p_{0}$ and $\rho=\rho_{0}$, corresponding to a fluid at rest at constant temperature, $T$, with uniform pressure, $p_{0}$, and density, $\rho_{0}$. The random stress tensor, $\mathbf{S}$, is of order $k_{\mathrm{B}} T$ and, consequently, macroscopically small. Thus, the corresponding fluctuations in the velocity, pressure and density fields are also macroscopically small. Therefore we introduce a linearized treatment of the Landau-Lifshitz equations, by setting $\mathbf{v}=\mathbf{v}^{(1)}, p=p_{0}+p^{(1)}$ and $\rho=\rho_{0}+\rho^{(1)}$, where the superscript (1) denotes a term of order $\mathbf{S}$.

We assume local equilibrium, which enables us to relate the density and pressure as

$$
p^{(1)}=c_{0}^{2} \rho^{(1)}, \quad \text { with } \quad c_{0}^{2}=\left(\frac{\partial p}{\partial \rho}\right)_{0} .
$$


Here $c_{0}$ is the adiabatic speed of sound, so that $\rho_{0} c_{0}^{2}$ equals the inverse adiabatic compressibility (NewtonLaplace equation). Eqs. (11) and (2) can be linearized as

$$
\begin{aligned}
& \eta \nabla^{2} \mathbf{v}^{(1)}+\left(\frac{\eta}{3}+\zeta\right) \nabla\left(\nabla \cdot \mathbf{v}^{(1)}\right) \\
& \quad-\nabla p^{(1)}-\rho_{0} \frac{\partial \mathbf{v}^{(1)}}{\partial t}=-\nabla \cdot \mathbf{S}, \\
& \frac{\partial \rho^{(1)}}{\partial t}+\rho_{0} \nabla \cdot \mathbf{v}^{(1)}=0
\end{aligned}
$$

or, in the frequency domain and using Eq. (9] [47],

$$
\begin{aligned}
& \eta \nabla^{2} \widetilde{\mathbf{v}}^{(1)}+\left(\frac{\eta}{3}+\zeta\right) \nabla\left(\nabla \cdot \widetilde{\mathbf{v}}^{(1)}\right) \\
& -c_{0}^{2} \nabla \widetilde{\rho}^{(1)}+i \omega \rho_{0} \widetilde{\mathbf{v}}^{(1)}=-\nabla \cdot \widetilde{\mathbf{S}}, \\
& \nabla \cdot \widetilde{\mathbf{v}}^{(1)}-\frac{i \omega}{\rho_{0}} \widetilde{\rho}^{(1)}=0 .
\end{aligned}
$$

We now introduce transverse and longitudinal components of the velocity fluctuations $\mathbf{v}^{(1)}$, which we denote $\mathbf{v}^{\mathrm{T}}$ and $\mathbf{v}^{\mathrm{L}}$, respectively. We have dropped the superscript (1) for notational simplicity, i.e., $\mathbf{v}^{(1)}=\mathbf{v}^{\mathrm{T}}+\mathbf{v}^{\mathrm{L}}$, with

$$
\nabla \cdot \mathbf{v}^{\mathrm{T}}=0 \quad \text { and } \quad \nabla \times \mathbf{v}^{\mathrm{L}}=0 .
$$

The random force density vector $\boldsymbol{\Sigma}=-\nabla \cdot \mathbf{S}$ can be decomposed into transverse and longitudinal components as well, using $\boldsymbol{\Sigma}=\boldsymbol{\Sigma}^{\mathrm{T}}+\boldsymbol{\Sigma}^{\mathrm{L}}$, where

$$
\nabla \cdot \boldsymbol{\Sigma}^{\mathrm{T}}=0 \quad \text { and } \quad \nabla \times \boldsymbol{\Sigma}^{\mathrm{L}}=0 .
$$

These random force density vector components have zero mean and zero cross correlations. Their self-correlations follow from Eq. (3) as

$$
\begin{aligned}
\left\langle\widetilde{\Sigma}_{i}^{\mathrm{L}}(\mathbf{r} ; \omega) \widetilde{\Sigma}_{j}^{\mathrm{L}}\left(\mathbf{r}^{\prime} ; \omega^{\prime}\right)\right\rangle=4 & \pi k_{\mathrm{B}} T\left(\frac{4 \eta}{3}+\zeta\right) \nabla_{i} \nabla_{j}^{\prime} \\
& \times \delta\left(\mathbf{r}-\mathbf{r}^{\prime}\right) \delta\left(\omega+\omega^{\prime}\right), \\
\left\langle\widetilde{\Sigma}_{i}^{\mathrm{T}}(\mathbf{r} ; \omega) \widetilde{\Sigma}_{j}^{\mathrm{T}}\left(\mathbf{r}^{\prime} ; \omega^{\prime}\right)\right\rangle=4 \pi & k_{\mathrm{B}} T \eta\left(\nabla_{k} \nabla_{k}^{\prime} \delta_{i j}-\nabla_{i} \nabla_{j}^{\prime}\right) \\
& \times \delta\left(\mathbf{r}-\mathbf{r}^{\prime}\right) \delta\left(\omega+\omega^{\prime}\right) .
\end{aligned}
$$

The stochastic Landau-Lifshitz equations can thus be written as

$$
\begin{aligned}
& \eta \nabla^{2} \widetilde{\mathbf{v}}^{\mathrm{L}}+\left(\frac{\eta}{3}+\zeta\right) \nabla\left(\nabla \cdot \widetilde{\mathbf{v}}^{\mathrm{L}}\right) \\
& -c_{0}^{2} \nabla \widetilde{\rho}^{(1)}+i \omega \rho_{0} \widetilde{\mathbf{v}}^{\mathrm{L}}=\widetilde{\boldsymbol{\Sigma}}^{\mathrm{L}}, \\
& \eta \nabla^{2} \widetilde{\mathbf{v}}^{\mathrm{T}}+i \omega \rho_{0} \widetilde{\mathbf{v}}^{\mathrm{T}}=\widetilde{\mathbf{\Sigma}}^{\mathrm{T}}, \\
& \nabla \cdot \widetilde{\mathbf{v}}^{\mathrm{L}}-\frac{i \omega}{\rho_{0}} \widetilde{\rho}^{(1)}=0 .
\end{aligned}
$$

We may simplify Eqs. (18)-(20) by using the vector identity

$$
\nabla_{j} \nabla_{j} \widetilde{v}_{i}^{\mathrm{L}}=\nabla_{j} \nabla_{j} \widetilde{v}_{i}^{\mathrm{L}}+\nabla_{j}\left(\nabla_{i} \widetilde{v}_{j}^{\mathrm{L}}-\nabla_{j} \widetilde{v}_{i}^{\mathrm{L}}\right)=\nabla_{i} \nabla_{j} \widetilde{v}_{j}^{\mathrm{L}}
$$

for the curl-free longitudinal component and by substituting Eq. (20) into Eq. (18) to obtain

$$
\begin{aligned}
& {\left[\frac{4 \eta}{3}+\zeta+\frac{i \rho_{0} c_{0}^{2}}{\omega}\right] \nabla^{2} \widetilde{\mathbf{v}}^{\mathrm{L}}+i \omega \rho_{0} \widetilde{\mathbf{v}}^{\mathrm{L}}=\widetilde{\mathbf{\Sigma}}^{\mathrm{L}},} \\
& \eta \nabla^{2} \widetilde{\mathbf{v}}^{\mathrm{T}}+i \omega \rho_{0} \widetilde{\mathbf{v}}^{\mathrm{T}}=\widetilde{\mathbf{\Sigma}}^{\mathrm{T}}
\end{aligned}
$$

We have now decoupled the transverse and longitudinal components of the velocity fluctuations. Eqs. (22) and (23) are nothing but the Langevin equations for each component of the velocity field in the frequency domain. In fact, Eq. (22) is a scalar equation for the longitudinal component of the velocity fluctuation [33].

The density field fluctuations can be obtained from the longitudinal component of the velocity field fluctuations,

$$
\widetilde{\rho}^{(1)}(\mathbf{r} ; \omega)=-\frac{i \rho_{0}}{\omega} \nabla \cdot \widetilde{\mathbf{v}}^{\mathrm{L}}(\mathbf{r} ; \omega) .
$$

\section{MEAN INTERACTION FORCE}

To obtain the net effective interaction force between the fluid's confining boundaries, we integrate the fluctuating hydrodynamic stress tensor, $\sigma_{i j}=\sigma_{i j}(\mathbf{r} ; t)$, over the bounding surfaces, $\Gamma$, i.e.,

$$
\left\langle\mathcal{F}_{i}(t)\right\rangle=\int_{\Gamma}\left\langle\sigma_{i j}(\mathbf{r} ; t)\right\rangle \mathrm{d} A_{j}
$$

where the fluctuating hydrodynamic stress tensor, which is 32

$$
\sigma_{i j}=\eta\left[\nabla_{i} v_{j}+\nabla_{j} v_{i}\right]-\left[\left(\frac{2 \eta}{3}-\zeta\right) \nabla_{k} v_{k}+p\right] \delta_{i j}+S_{i j}
$$

can be written up to first order in the field fluctuations as $\sigma_{i j}=-p_{0} \delta_{i j}+\sigma_{i j}^{(1)}$, with

$$
\begin{aligned}
\sigma_{i j}^{(1)}=\eta[ & \left.\nabla_{i} v_{j}^{(1)}+\nabla_{j} v_{i}^{(1)}\right] \\
& -\left[\left(\frac{2 \eta}{3}-\zeta\right) \nabla_{k} v_{k}^{(1)}+c_{0}^{2} \rho^{(1)}\right] \delta_{i j}+S_{i j} .
\end{aligned}
$$

The stress tensor is linear in the fluid fluctuations, which are themselves linear in the random stress tensor and, thus, their ensemble averages vanish $\left\langle\mathbf{v}^{\mathrm{T}}(\mathbf{r} ; t)\right\rangle=$ $\left\langle\mathbf{v}^{\mathrm{L}}(\mathbf{r} ; t)\right\rangle=0$ and $\left\langle\rho^{(1)}(\mathbf{r} ; t)\right\rangle=0$. As a result, at first order in field fluctuations, the net fluctuation-induced force acting on the fluid boundaries must vanish, irrespective of the geometry of the fluid system, i.e.,

$$
\left\langle\mathcal{F}_{i}^{(1)}(t)\right\rangle=0 \text {. }
$$

We note that the mean force at leading order stems from the equilibrium pressure and is simply $\mathcal{F}_{z}^{(0)}=-p_{0} A$. We exclude this contribution in the rest of our discussion and focus on the statistical properties of the force at first order in the field fluctuations. 
In what follows, we limit our discussion to the planeparallel geometry of two rigid walls of arbitrarily large surface area, $A$. We assume that the walls are located along the $z$ axis at $z=0$ and $z=L$ at a separation distance of $L$ and that the fluid velocity satisfies no-slip boundary conditions on the walls.

\section{TWO-POINT, TIME-DEPENDENT CORRELATIONS OF THE FORCE}

Although, as we have already noted, the mean interplate force due to hydrodynamic fluctuations in the fluid layer must vanish, its variance or correlation functions need not and do not. In this Section, we study the twopoint, time-dependent correlators, including the variance, of the forces that act on the boundaries in the two-wall geometry. In this plane-parallel geometry, we are primarily concerned with the force perpendicular to the plane boundaries, in which case the two-point, timedependent force correlator is given by

$$
\begin{aligned}
& \mathcal{C}\left(z, z^{\prime} ; t, t^{\prime}\right)=\left\langle\mathcal{F}_{z}^{(1)}(z ; t) \mathcal{F}_{z}^{(1)}\left(z^{\prime} ; t^{\prime}\right)\right\rangle \\
& \quad=\iint_{A}\left\langle\sigma_{z z}(\mathbf{r} ; t) \sigma_{z z}\left(\mathbf{r}^{\prime} ; t^{\prime}\right)\right\rangle \mathrm{d} x \mathrm{~d} y \mathrm{~d} x^{\prime} \mathrm{d} y^{\prime},
\end{aligned}
$$

where the integrals run over the surface areas $A$ of the two walls that are located at $z=0$ and $z=L$. Throughout this paper, we use an uppercase $\mathcal{C}$ to denote correlation functions of the normal forces acting on the fluid boundaries and a lowercase $c$ to refer to correlation functions of the fluctuating hydrodynamic fields. We express the former quantity in terms of the latter ones (see Appendix A). In the present case, the correlators of the velocity and density fluctuations are given by

$$
\begin{aligned}
c_{i j}^{\mathrm{TT}}\left(\mathbf{r}, \mathbf{r}^{\prime} ; t, t^{\prime}\right) & =\left\langle v_{i}^{\mathrm{T}}(\mathbf{r} ; t) v_{j}^{\mathrm{T}}\left(\mathbf{r}^{\prime} ; t^{\prime}\right)\right\rangle, \\
c_{i j}^{\mathrm{LL}}\left(\mathbf{r}, \mathbf{r}^{\prime} ; t, t^{\prime}\right) & =\left\langle v_{i}^{\mathrm{L}}(\mathbf{r} ; t) v_{j}^{\mathrm{L}}\left(\mathbf{r}^{\prime} ; t^{\prime}\right)\right\rangle, \\
c^{\rho \rho}\left(\mathbf{r}, \mathbf{r}^{\prime} ; t, t^{\prime}\right) & =\left\langle\rho^{(1)}(\mathbf{r} ; t) \rho^{(1)}\left(\mathbf{r}^{\prime} ; t^{\prime}\right)\right\rangle .
\end{aligned}
$$

The cross-correlation function of the transverse and longitudinal components of the velocity vanishes by construction. Furthermore, the transverse velocity and density fluctuations are independent fields, with vanishing cross-correlation function. Therefore, the only other correlation function we need is the density-velocity crosscorrelator,

$$
c_{i}^{\mathrm{L} \rho}\left(\mathbf{r}, \mathbf{r}^{\prime} ; t, t^{\prime}\right)=\left\langle v_{i}^{\mathrm{L}}(\mathbf{r} ; t) \rho^{(1)}\left(\mathbf{r}^{\prime} ; t^{\prime}\right)\right\rangle .
$$

Not all of these correlators contribute to the timedependent correlator of the forces between the two hard boundaries. In Appendices $\mathrm{B}$ and $\mathrm{D}$. we show that the contributions to the normal force correlator generated by the correlation function of the transverse velocity field and by the correlation function between the velocity and density fields vanish for our geometry. Therefore, applying the formulae of the previous Section, we can write the time-dependent force correlator as the sum of three terms (see Appendix $\mathrm{A}$ for details),

$$
\mathcal{C}\left(z, z^{\prime} ; t, t^{\prime}\right)=\sum_{i=0}^{2} \mathcal{P}_{i}\left(z, z^{\prime} ; t, t^{\prime}\right)
$$

Defining the dimensionless parameter

$$
\chi=4 / 3+\zeta / \eta
$$

we can write the first term as

$$
\mathcal{P}_{0}\left(z, z^{\prime} ; t, t^{\prime}\right) \equiv 2 k_{\mathrm{B}} T \eta \chi A \delta\left(z-z^{\prime}\right) \delta\left(t-t^{\prime}\right) .
$$

This contribution stems directly from the integration of the random stress correlator, $\left\langle S_{z z}(\mathbf{r} ; t) S_{z z}\left(\mathbf{r}^{\prime} ; t^{\prime}\right)\right\rangle$, over the bounding surfaces; this term vanishes unless $z=z^{\prime}$ and $t=t^{\prime}$, in which case it reduces to an irrelevant constant that will be dropped in the rest of our analysis. The two other terms are

$$
\begin{aligned}
& \mathcal{P}_{1}\left(z, z^{\prime} ; t, t^{\prime}\right) \equiv\left(\frac{4 \eta}{3}+\zeta\right)^{2} \iint_{A} \mathrm{~d} x \mathrm{~d} y \mathrm{~d} x^{\prime} \mathrm{d} y^{\prime} \\
& \times \nabla_{z} \nabla_{z}^{\prime} c_{z z}^{\mathrm{LL}}\left(\mathbf{r}, \mathbf{r}^{\prime} ; t, t^{\prime}\right), \\
& \mathcal{P}_{2}\left(z, z^{\prime} ; t, t^{\prime}\right) \equiv c_{0}^{4} \iint_{A} \mathrm{~d} x \mathrm{~d} y \mathrm{~d} x^{\prime} \mathrm{d} y^{\prime} c^{\rho \rho}\left(\mathbf{r}, \mathbf{r}^{\prime} ; t, t^{\prime}\right) .
\end{aligned}
$$

We note that, in the above, we have used Eq. (24), which relates the density fluctuations to the fluctuations of the longitudinal components of the velocity.

With this expression in hand, we can see that we need to determine the correlation functions of the density fields and the longitudinal component of the velocity fields. We proceed via the following steps [32]:

1. Obtain the Green functions of Eq. (22);

2. Express the fluctuating fields and their correlation functions in terms of the Green functions above;

3. Integrate the resulting expressions over the boundaries of the fluid according to Eqs. (37) and (38).

\section{A. Green functions}

In the present model with no-slip walls, the velocity and, therefore, the corresponding Green function should vanish at the boundaries. Translational invariance in the two (transverse) directions perpendicular to the $z$-axis prompts us to search for Green functions of the form

$$
\widetilde{G}\left(\mathbf{r}, \mathbf{r}^{\prime \prime} ; \omega\right)=\frac{1}{(2 \pi)^{2}} \int \mathrm{d}^{2} \mathbf{k} e^{i \mathbf{k} \cdot\left(\mathbf{s}-\mathbf{s}^{\prime \prime}\right)} \widetilde{G}\left(z, z^{\prime \prime} ; \mathbf{k} ; \omega\right),
$$

where $\mathbf{r}=(\mathbf{s}, z)$, with $\mathbf{s}=(x, y)$, and $\mathbf{k}=\left(k_{x}, k_{y}\right)$. The longitudinal Green function corresponding to Eq. (22) is a solution of the following equation:

$$
\left[\nabla_{z}^{2}-m^{2}\right] \widetilde{G}^{\mathrm{L}}\left(z, z^{\prime \prime} ; \mathbf{k} ; \omega\right)=\frac{i \lambda^{2}}{\omega \rho_{0}} \delta\left(z-z^{\prime \prime}\right),
$$


where $m^{2}=\mathbf{k}^{2}+\lambda^{2}$ and we have defined the longitudinal decay constant $\lambda$ as

$$
\lambda^{2}=-\frac{i \omega^{2} \rho_{0}}{(4 \eta / 3+\zeta) \omega+i \rho_{0} c_{0}^{2}} .
$$

The solution of Eq. (40) is well known [48 50], and with no-slip boundary conditions at $z=0$ and $z=L$, the Green function is obtained as

$$
\begin{aligned}
\widetilde{G}^{\mathrm{L}}\left(z, z^{\prime \prime} ; \mathbf{k} ; \omega\right)=g_{1}^{\mathrm{L}} e^{-m z}+g_{2}^{\mathrm{L}} e^{m(z-L)} & \\
& -\frac{i \lambda^{2}}{2 m \omega \rho_{0}} e^{-m\left|z-z^{\prime \prime}\right|},
\end{aligned}
$$

where

$$
\begin{aligned}
& g_{1}^{\mathrm{L}}=\frac{i \lambda^{2}}{2 m \omega \rho_{0}} \operatorname{csch}(m L) \sinh \left(m\left(L-z^{\prime \prime}\right)\right), \\
& g_{2}^{\mathrm{L}}=\frac{i \lambda^{2}}{2 m \omega \rho_{0}} \operatorname{csch}(m L) \sinh \left(m z^{\prime \prime}\right),
\end{aligned}
$$

are constants of integration that satisfy the no-slip boundary conditions.

\section{B. Characteristic scales and dimensionless parameters}

We simplify the following analysis by introducing dimensionless parameters that characterize the fluid and the plane-parallel geometry of our system. There are two length scales that can be used for this purpose: The macroscopic plate separation, $L$, and the microscopic scale at which the continuum hydrodynamic description breaks down, which we denote $a$. There are two characteristic vorticity frequencies associated with each of these length scales [48],

$$
\omega_{0}=\frac{\eta}{L^{2} \rho_{0}} \quad \text { and } \quad \omega_{\infty}=\frac{\eta}{a^{2} \rho_{0}} .
$$

The inverse frequencies, $\omega_{0}^{-1}$ and $\omega_{\infty}^{-1}$, correspond to the time that vorticity requires to diffuse a certain distance, in this case $L$ or $a$, respectively. We also define the dimensionless parameter $\gamma$, which is given by

$$
\gamma=\frac{c_{0}^{2}}{L^{2} \omega_{0}^{2}}=\left(\frac{L \rho_{0} c_{0}}{\eta}\right)^{2} \text {. }
$$

This parameter is the squared ratio of the vorticity time scale and the typical compression time scale in which a propagating sound wave travels a distance $L$ [48].

To facilitate our later discussions, we introduce the dimensionless ratios

$$
u=\frac{\omega}{\omega_{0} \gamma} \quad \text { and } \quad u_{\infty}=\frac{\omega_{\infty}}{\omega_{0} \gamma},
$$

and define the function

$$
f_{m}(u)=\frac{u^{2-m}}{1+\chi^{2} u^{2}} .
$$

We can now express the real and imaginary parts of the longitudinal decay constant, $\lambda$, as

$$
\begin{aligned}
& \ell_{+}=\lambda_{\mathrm{R}} L=\frac{\omega_{0} \gamma L}{c_{0}} \frac{|u|}{\sqrt{2}} \sqrt{\left[1-\sqrt{f_{2}(u)}\right] \sqrt{f_{2}(u)}}, \\
& \ell_{-}=\lambda_{\mathrm{I}} L=-\frac{\omega_{0} \gamma L}{c_{0}} \frac{u}{\sqrt{2}} \sqrt{\left[1+\sqrt{f_{2}(u)}\right] \sqrt{f_{2}(u)}} .
\end{aligned}
$$

The vorticity frequency scale $\omega_{0}$ marks the boundary between the low-frequency propagative regime, for which $\omega<\omega_{0} \gamma$ (or $u<1$ ) and sound waves propagate with speed $c \sim\left|\lambda_{\mathrm{I}}^{-1}\right|$, and the high-frequency diffusive regime, for which $\omega>\omega_{0} \gamma$ (or $u>1$ ) and viscosity effects damp compression perturbations [48]. Furthermore, the dimensionless ratio $u_{\infty}$ can be expressed in terms of a new length scale $\delta$ :

$$
u_{\infty}=\frac{\delta^{2}}{a^{2}} \quad \text { where } \quad \delta=\frac{\eta}{\rho_{0} c_{0}}=\frac{c_{0}}{\omega_{0} \gamma} .
$$

This length scale characterizes the boundary between the propagative and diffusive regimes at $\omega_{0} \gamma$. We can also define a characteristic time scale,

$$
t_{0}=\delta / c_{0},
$$

associated with this boundary. Finally, then, we can write $\ell_{+}$and $\ell_{-}$as

$$
\begin{aligned}
& \ell_{+}=\frac{L}{\delta} \frac{|u|}{\sqrt{2}} \sqrt{\left[1-\sqrt{f_{2}(u)}\right] \sqrt{f_{2}(u)}}, \\
& \ell_{-}=-\frac{L}{\delta} \frac{u}{\sqrt{2}} \sqrt{\left[1+\sqrt{f_{2}(u)}\right] \sqrt{f_{2}(u)}} .
\end{aligned}
$$

For any reasonable choice of realistic parameters for a fluid far from the critical point, we have $u \ll 1$, i.e., we work in the propagative regime. In this case, the plate separation of a realistic experiment satisfies $L / \delta \gg 1$. For liquids close to the critical point, or polymers in solution, however, the crossover frequency can be much lower and, therefore, we can have $u \gg 1$. In this case, the system is in the diffusive regime and the crossover length scale, $\delta$, may be macroscopic.

\section{Correlation functions}

Now that we have explicit expressions for the Green function solutions in hand, we turn to the correlation functions $c_{z z}^{\mathrm{LL}}$ and $c^{\rho \rho}$, which enter in Eqs. (34)-(38), and express these correlation functions in terms of the corresponding Green functions. Here, we simply sketch the derivation for $c_{z z}^{\mathrm{LL}}$, as an example, and leave the details of the corresponding calculation of $c^{\rho \rho}$ to Appendix C.

The longitudinal velocity fluctuations are given in terms of the longitudinal Green function as

$$
v_{i}^{\mathrm{L}}(\mathbf{r} ; t)=\int \mathrm{d} t^{\prime \prime} \int \mathrm{d}^{3} \mathbf{r}^{\prime \prime} G^{\mathrm{L}}\left(\mathbf{r}, \mathbf{r}^{\prime \prime} ; t-t^{\prime \prime}\right) \Sigma_{i}^{\mathrm{L}}\left(\mathbf{r}^{\prime \prime} ; t^{\prime \prime}\right) .
$$


We require the correlation function

$$
\begin{aligned}
\left\langle v_{i}^{\mathrm{L}}(\mathbf{r} ; t)\right. & \left.v_{j}^{\mathrm{L}}\left(\mathbf{r}^{\prime} ; t^{\prime}\right)\right\rangle=\int \mathrm{d} t^{\prime \prime} \int \mathrm{d} t^{\prime \prime \prime} \int \mathrm{d}^{3} \mathbf{r}^{\prime \prime} \int \mathrm{d}^{3} \mathbf{r}^{\prime \prime \prime} \\
& \times G^{\mathrm{L}}\left(\mathbf{r}, \mathbf{r}^{\prime \prime} ; t-t^{\prime \prime}\right) G^{\mathrm{L}}\left(\mathbf{r}^{\prime}, \mathbf{r}^{\prime \prime \prime} ; t^{\prime}-t^{\prime \prime \prime}\right) \\
& \times\left\langle\Sigma_{i}^{\mathrm{L}}\left(\mathbf{r}^{\prime \prime} ; t^{\prime \prime}\right) \Sigma_{j}^{\mathrm{L}}\left(\mathbf{r}^{\prime \prime \prime} ; t^{\prime \prime \prime}\right)\right\rangle
\end{aligned}
$$

Recalling the stochastic properties of the random stress tensor, Eq. (16), we obtain

$$
\begin{aligned}
& c_{i j}^{\mathrm{LL}}\left(\mathbf{r}, \mathbf{r}^{\prime} ; t, t^{\prime}\right)=2 k_{\mathrm{B}} T \eta \chi \int \mathrm{d} t^{\prime \prime} \int \mathrm{d}^{3} \mathbf{r}^{\prime \prime} \\
& \quad \times \nabla_{i}^{\prime \prime} G^{\mathrm{L}}\left(\mathbf{r}, \mathbf{r}^{\prime \prime} ; t-t^{\prime \prime}\right) \nabla_{j}^{\prime \prime} G^{\mathrm{L}}\left(\mathbf{r}^{\prime}, \mathbf{r}^{\prime \prime} ; t^{\prime}-t^{\prime \prime}\right) .
\end{aligned}
$$

We now introduce a Fourier representation of the Green functions

$$
\begin{aligned}
& c_{i j}^{\mathrm{LL}}\left(\mathbf{r}, \mathbf{r}^{\prime} ;\right.\left.t, t^{\prime}\right)=2 k_{\mathrm{B}} T \eta \chi \int \mathrm{d} t^{\prime \prime} \int \mathrm{d}^{3} \mathbf{r}^{\prime \prime} \\
& \times \int \frac{\mathrm{d} \omega}{2 \pi} e^{-i \omega\left(t-t^{\prime \prime}\right)} \nabla_{i}^{\prime \prime} \widetilde{G}^{\mathrm{L}}\left(\mathbf{r}, \mathbf{r}^{\prime \prime} ; \omega\right) \\
& \times \int \frac{\mathrm{d} \omega^{\prime}}{2 \pi} e^{-i \omega^{\prime}\left(t^{\prime}-t^{\prime \prime}\right)} \nabla_{j}^{\prime \prime} \widetilde{G}^{\mathrm{L}}\left(\mathbf{r}^{\prime}, \mathbf{r}^{\prime \prime} ; \omega^{\prime}\right) .
\end{aligned}
$$

The integral over $t^{\prime \prime}$ generates a Dirac delta function for the frequencies, $\delta\left(\omega+\omega^{\prime}\right)$, and therefore one of the frequency integrals is trivial:

$$
\begin{aligned}
& c_{i j}^{\mathrm{LL}}\left(\mathbf{r}, \mathbf{r}^{\prime} ; t, t^{\prime}\right)=2 k_{\mathrm{B}} T \eta \chi \int \frac{\mathrm{d} \omega^{\prime}}{2 \pi} e^{i \omega^{\prime}\left(t-t^{\prime}\right)} \\
& \quad \times \int \mathrm{d}^{3} \mathbf{r}^{\prime \prime} \nabla_{i}^{\prime \prime} \widetilde{G}^{\mathrm{L}}\left(\mathbf{r}, \mathbf{r}^{\prime \prime} ;-\omega^{\prime}\right) \nabla_{j}^{\prime \prime} \widetilde{G}^{\mathrm{L}}\left(\mathbf{r}^{\prime}, \mathbf{r}^{\prime \prime} ; \omega^{\prime}\right) .
\end{aligned}
$$

In principle, we could substitute our explicit expression for the Green function, Eq. (42), into this correlation function and attempt to directly calculate the integrals at this stage. We will see, however, that this is not the most straightforward approach: Spatial integrations over the fluid boundary will simplify our task considerably. We also take advantage of the fact that we only require the components of the velocity fields perpendicular to the plane boundaries. Therefore, we set $i=j=z$ in our expression for the correlation function, $c_{i j}^{\mathrm{LL}}\left(\mathbf{r}, \mathbf{r}^{\prime} ; t, t^{\prime}\right)$, and use the translational-invariant structure of the Green function, Eq. (39), to write

$$
\begin{aligned}
& c_{z z}^{\mathrm{LL}}\left(\mathbf{r}, \mathbf{r}^{\prime} ; t, t^{\prime}\right)=2 k_{\mathrm{B}} T \eta \chi \int \frac{\mathrm{d} \omega^{\prime}}{2 \pi} e^{i \omega^{\prime}\left(t-t^{\prime}\right)} \\
& \quad \times \int \mathrm{d}^{3} \mathbf{r}^{\prime \prime} \int \frac{\mathrm{d}^{2} \mathbf{k}}{(2 \pi)^{2}} e^{i \mathbf{k} \cdot\left(\mathbf{s}-\mathbf{s}^{\prime \prime}\right)} \nabla_{z}^{\prime \prime} \widetilde{G}^{\mathrm{L}}\left(z, z^{\prime \prime} ; \mathbf{k} ;-\omega^{\prime}\right) \\
& \quad \times \int \frac{\mathrm{d}^{2} \mathbf{k}^{\prime}}{(2 \pi)^{2}} e^{i \mathbf{k}^{\prime} \cdot\left(\mathbf{s}^{\prime}-\mathbf{s}^{\prime \prime}\right)} \nabla_{z}^{\prime \prime} \widetilde{G}^{\mathrm{L}}\left(z^{\prime}, z^{\prime \prime} ; \mathbf{k}^{\prime} ; \omega^{\prime}\right) .
\end{aligned}
$$

The double integral over $\mathbf{s}^{\prime \prime}$ generates a wavenumber Dirac delta function, $\delta\left(\mathbf{k}+\mathbf{k}^{\prime}\right)$, that enables us to carry out one of the wavenumber integrals immediately and, thus, obtain

$$
\begin{aligned}
& c_{z z}^{\mathrm{LL}}\left(\mathbf{r}, \mathbf{r}^{\prime} ; t, t^{\prime}\right)=2 k_{\mathrm{B}} T \eta \chi \int \frac{\mathrm{d} \omega^{\prime}}{2 \pi} e^{i \omega^{\prime}\left(t-t^{\prime}\right)} \\
& \times \int \mathrm{d} z^{\prime \prime} \int \frac{\mathrm{d}^{2} \mathbf{k}}{(2 \pi)^{2}} e^{i \mathbf{k} \cdot\left(\mathbf{s}-\mathbf{s}^{\prime}\right)} \nabla_{z}^{\prime \prime} \widetilde{G}^{\mathrm{L}}\left(z, z^{\prime \prime} ; \mathbf{k} ;-\omega^{\prime}\right) \\
& \times \nabla_{z}^{\prime \prime} \widetilde{G}^{\mathrm{L}}\left(z^{\prime}, z^{\prime \prime} ;-\mathbf{k} ; \omega^{\prime}\right) .
\end{aligned}
$$

Analogous arguments apply to the density correlation function, which is (see Appendix $\mathrm{C}$ )

$$
\begin{aligned}
& c^{\rho \rho}\left(\mathbf{r}, \mathbf{r}^{\prime} ; t, t^{\prime}\right)=\frac{k_{\mathrm{B}} T}{\pi} \rho_{0}^{2} \eta \chi \int \frac{\mathrm{d} \omega^{\prime}}{\omega^{\prime 2}} e^{i \omega^{\prime}\left(t-t^{\prime}\right)} \int \mathrm{d} z^{\prime \prime} \\
& \quad \times \int \frac{\mathrm{d}^{2} \mathbf{k}}{(2 \pi)^{2}} e^{i \mathbf{k} \cdot\left(\mathbf{s}-\mathbf{s}^{\prime}\right)}\left(\nabla_{z} \nabla_{z}^{\prime \prime}+\mathbf{k}^{2}\right) \widetilde{G}^{\mathrm{L}}\left(z, z^{\prime \prime} ; \mathbf{k} ;-\omega^{\prime}\right) \\
& \quad \times\left(\nabla_{z}^{\prime} \nabla_{z}^{\prime \prime}+\mathbf{k}^{2}\right) \widetilde{G}^{\mathrm{L}}\left(z^{\prime}, z^{\prime \prime} ;-\mathbf{k} ; \omega^{\prime}\right) .
\end{aligned}
$$

\section{Spatial integration over surface boundaries}

Our final step is to integrate the correlation functions, Eqs. (61) and (62), over the boundaries of the fluid according to Eqs. (34)-(38). These integrals give our final result for the time-dependent correlators of the force acting on the fluid boundaries.

The double integrals over $(x, y)$ and $\left(x^{\prime}, y^{\prime}\right)$ in Eqs. (34)-38) lead to a Dirac delta function over the transverse wavenumbers, $(2 \pi)^{2} A \delta(\mathbf{k})$. Thus, we can write these equations in terms of the Green function as

$$
\begin{aligned}
& \mathcal{P}_{1}\left(z, z^{\prime} ; t, t^{\prime}\right)=\frac{k_{\mathrm{B}} T}{\pi} \eta^{3} \chi^{3} A \int \mathrm{d} \omega^{\prime} \cos \left[\omega^{\prime}\left(t-t^{\prime}\right)\right] \\
& \times \int \mathrm{d} z^{\prime \prime} \nabla_{z} \nabla_{z}^{\prime \prime} \widetilde{G}^{\mathrm{L}}\left(z, z^{\prime \prime} ; \mathbf{0} ;-\omega^{\prime}\right) \nabla_{z}^{\prime} \nabla_{z}^{\prime \prime} \widetilde{G}^{\mathrm{L}}\left(z^{\prime}, z^{\prime \prime} ; \mathbf{0} ; \omega^{\prime}\right), \\
& \mathcal{P}_{2}\left(z, z^{\prime} ; t, t^{\prime}\right)=\frac{k_{\mathrm{B}} T}{\pi} \rho_{0}^{2} \eta \chi c_{0}^{4} A \int \frac{\mathrm{d} \omega^{\prime}}{\omega^{\prime 2}} \cos \left[\omega^{\prime}\left(t-t^{\prime}\right)\right] \\
& \times \int \mathrm{d} z^{\prime \prime} \nabla_{z} \nabla_{z}^{\prime \prime} \widetilde{G}^{\mathrm{L}}\left(z, z^{\prime \prime} ; \mathbf{0} ;-\omega^{\prime}\right) \nabla_{z}^{\prime} \nabla_{z}^{\prime \prime} \widetilde{G}^{\mathrm{L}}\left(z^{\prime}, z^{\prime \prime} ; \mathbf{0} ; \omega^{\prime}\right) .
\end{aligned}
$$

These frequency integrals run over the frequency range $\omega \in\left[-\omega_{\infty}, \omega_{\infty}\right]$ and the spatial integral is over $z \in[0, L]$. In writing the above relations, we have used the fact that the integrands involved in calculating $\mathcal{P}_{1}$ and $\mathcal{P}_{2}$ (see Eqs. (61) and (62) ) have odd imaginary parts, which thus vanish, leading to the factor $\cos \left[\omega^{\prime}\left(t-t^{\prime}\right)\right]$ from the real part of the exponential factor $e^{i \omega^{\prime}\left(t-t^{\prime}\right)}$. We also note that $\widetilde{G}^{\mathrm{L} *}\left(z^{\prime}, z^{\prime \prime} ; \mathbf{0} ; \omega^{\prime}\right)=\widetilde{G}^{\mathrm{L}}\left(z^{\prime}, z^{\prime \prime} ; \mathbf{0} ;-\omega^{\prime}\right)$, which follows from the reality of $G^{\mathrm{L}}\left(z^{\prime}, z^{\prime \prime} ; \mathbf{0} ; t\right)$. Therefore, as expected, the final correlators are purely real.

Carrying out the derivatives and the remaining spatial 
integral is fairly straightforward. The results are

$$
\begin{aligned}
& \mathcal{P}_{1}\left(z, z^{\prime} ; t, t^{\prime}\right)=\frac{k_{\mathrm{B}} T}{\pi} \frac{\rho_{0} c_{0}^{2} A}{L} \chi^{3}\left[\mathcal{W}_{0}\left(z, z^{\prime} ; \tau\right)\right. \\
& \left.\quad+L \mathcal{V}_{0}\left(z, z^{\prime} ; \tau\right) \delta\left(z-z^{\prime}\right)\right] \\
& \begin{aligned}
\mathcal{P}_{2}\left(z, z^{\prime} ; t, t^{\prime}\right)=\frac{k_{\mathrm{B}} T}{\pi} \frac{\rho_{0} c_{0}^{2} A}{L} \chi\left[\mathcal{W}_{2}\left(z, z^{\prime} ; \tau\right)\right. \\
\left.+L \mathcal{V}_{2}\left(z, z^{\prime} ; \tau\right) \delta\left(z-z^{\prime}\right)\right]
\end{aligned}
\end{aligned}
$$

The relevant frequency integrals are given by (see Appendix E)

$$
\begin{aligned}
& \mathcal{W}_{m}(0,0 ; \tau)=2 \int_{0}^{u_{\infty}} \mathrm{d} u f_{m}(u) \cos [u \tau] \\
& \times \frac{1}{\cosh \left[2 \ell_{+}\right]-\cos \left[2 \ell_{-}\right]}\left[\left(\frac{\ell^{2}}{2 \ell_{-}}-2 \ell_{-}\right) \sin \left[2 \ell_{-}\right]\right. \\
& \left.\quad+\left(\frac{\ell^{2}}{2 \ell_{+}}-2 \ell_{+}\right) \sinh \left[2 \ell_{+}\right]\right], \\
& \mathcal{W}_{m}(0, L ; \tau)=2 \int_{0}^{u_{\infty}} \mathrm{d} u f_{m}(u) \cos [u \tau] \\
& \times \frac{1}{\cosh \left[2 \ell_{+}\right]-\cos \left[2 \ell_{-}\right]}\left[\left(\frac{\ell^{2}}{\ell_{-}}-4 \ell_{-}\right) \cosh \left[\ell_{+}\right] \sin \left[\ell_{-}\right]\right. \\
& \left.\quad+\left(\frac{\ell^{2}}{\ell_{+}}-4 \ell_{+}\right) \cos \left[\ell_{-}\right] \sinh \left[\ell_{+}\right]\right]
\end{aligned}
$$

and

$$
\mathcal{V}_{m}(0,0 ; \tau)=2 \int_{0}^{u_{\infty}} \mathrm{d} u f_{m}(u) \cos [u \tau] .
$$

In these equations $\ell^{2}=\ell_{+}^{2}+\ell_{-}^{2}$, where we have defined $\ell_{+}$and $\ell_{-}$in Eqs. (53) and (54) and the function $f_{m}(u)$ in Eq. (48). The dimensionless time parameter is

$$
\tau=\left(t-t^{\prime}\right) / t_{0},
$$

with $t_{0}$ the characteristic microscopic timescale defined in Eq. (52). We have used the symmetry of the integrand to integrate over the positive real axis up to the dimensionless microscopic cutoff, $u_{\infty}$, of Eqs. (47) and (51). To simplify these expressions further, we note that

$$
\mathcal{V}_{2}(0,0 ; \tau)+\chi^{2} \mathcal{V}_{0}(0,0 ; \tau)=\frac{2}{\tau} \sin \left[u_{\infty} \tau\right]
$$

Putting together all of these results, from Eqs. (34) and (63)-(71), we find

$$
\begin{aligned}
\mathcal{C}\left(0,0 ; t, t^{\prime}\right)=\frac{k_{\mathrm{B}} T}{\pi} & \frac{\rho_{0} c_{0}^{2} A}{L} \chi\left[\frac{2 L}{\tau} \sin \left[u_{\infty} \tau\right] \delta(0)\right. \\
& \left.+\chi^{2} \mathcal{W}_{0}(0,0 ; \tau)+\mathcal{W}_{2}(0,0 ; \tau)\right], \\
\mathcal{C}\left(0, L ; t, t^{\prime}\right)= & \frac{k_{\mathrm{B}} T}{\pi} \frac{\rho_{0} c_{0}^{2} A}{L} \chi \\
& \times\left[\chi^{2} \mathcal{W}_{0}(0, L ; \tau)+\mathcal{W}_{2}(0, L ; \tau)\right] .
\end{aligned}
$$

These are our final results: The same-plate and the cross-plate correlators of the normal force, expressed in terms of the four frequency integrals $\mathcal{W}_{m}(0,0 ; \tau)$ and $\mathcal{W}_{m}(0, L ; \tau)$ where $m=0,2$. Thus, while the average fluctuation-induced force between the bounding surfaces vanishes identically (see Sec. III), the correlation functions of the force show a pronounced dependence on the inter-plate separation and the time difference.

The same-plate correlator of the normal force, $\mathcal{C}\left(0,0 ; t, t^{\prime}\right)$ in Eq. (72), contains three terms. The first contribution is local in space and therefore proportional to the Dirac delta function. Comparing this first term to $\mathcal{P}_{0}\left(z, z^{\prime} ; t, t^{\prime}\right)$ in Eq. (36) indicates that this contribution to $\mathcal{C}\left(0,0 ; t, t^{\prime}\right)$ is related to the integral of the random stress correlator, Eq. (3), across the bounding surfaces, but with the hydrodynamic coupling nevertheless fully taken into account. Incorporating the hydrodynamic coupling leads to non-locality in time, while locality in space is preserved at leading order. In contrast, $\mathcal{P}_{0}\left(z, z^{\prime} ; t, t^{\prime}\right)$ is local both in time and in space, because this term follows directly from the correlator of the random stress tensor without any hydrodynamic coupling and has, therefore, been dropped from our present analysis. The other two terms in $\mathcal{C}\left(0,0 ; t, t^{\prime}\right)$ are different in nature. They are non-local both in time and in space. They correspond to self-correlations mediated by the hydrodynamic interaction between the boundaries, leading to separation-dependent contributions to the same-plate, normal force correlator. These two terms present a nontrivial generalization of the normal force correlator that hydrodynamically couples the boundaries. We now define these contributions to be the excess correlator,

$$
\begin{aligned}
\Delta \mathcal{C}\left(0,0 ; t, t^{\prime}\right) \equiv & \frac{k_{\mathrm{B}} T}{\pi} \frac{\rho_{0} c_{0}^{2} A}{L} \chi \\
& \times\left[\chi^{2} \mathcal{W}_{0}(0,0 ; \tau)+\mathcal{W}_{2}(0,0 ; \tau)\right],
\end{aligned}
$$

which we investigate in detail in the following sections.

The cross-plate correlator of the normal force in Eq. (73) does not contain any local terms. In fact, it is purely non-local and does not include any hydrodynamic self-interactions. The cross-plate correlator is due entirely to hydrodynamic interactions across the fluid between the boundaries, and thus naturally depends on the boundary separation.

In summary, for the same-plate force correlator, we have identified a trivial term that is local in space and non-trivial terms that are non-local in space and correspond to self-correlations mediated by the hydrodynamic coupling between the boundaries. This leads to the separation-dependent excess same-plate force correlator. On the other hand, the cross-plate force correlator contains no local terms, as expected, and stems entirely from hydrodynamic interactions between the bounding surfaces. 
TABLE I. Representative ranges of physical parameters in a realistic fluid; see the text for definitions.

\begin{tabular}{ccc}
\hline \hline Parameter & Description & Range \\
\hline$L$ & Plate separation & $10^{-6}$ to $10^{-3} \mathrm{~m}$ \\
$\delta$ & Propagative-diffusive boundary & $10^{-9}$ to $10^{-6} \mathrm{~m}$ \\
$a$ & Microscopic cutoff & $10^{-9} \mathrm{~m}$ \\
$\eta$ & Shear viscosity & $10^{-4}$ to $1 \mathrm{~Pa} \cdot \mathrm{s}$ \\
$\zeta$ & Bulk viscosity & $10^{-4}$ to $1 \mathrm{~Pa} \cdot \mathrm{s}$ \\
\hline \hline
\end{tabular}

\section{RESULTS FOR EQUAL-TIME FORCE CORRELATORS}

Our task now is to explore and evaluate the frequency integrals that appear in Eqs. (72)-(74). We start by considering the equal-time correlators that follow from these equations by setting $\tau=0$ or, equivalently, $t=t^{\prime}$, i.e.,

$$
\begin{aligned}
\Delta \mathcal{C}(0,0) & =\frac{k_{\mathrm{B}} T}{\pi} \frac{\rho_{0} c_{0}^{2} A}{L} \chi\left[\chi^{2} \mathcal{W}_{0}(0,0)+\mathcal{W}_{2}(0,0)\right], \\
\mathcal{C}(0, L) & =\frac{k_{\mathrm{B}} T}{\pi} \frac{\rho_{0} c_{0}^{2} A}{L} \chi\left[\chi^{2} \mathcal{W}_{0}(0, L)+\mathcal{W}_{2}(0, L)\right]
\end{aligned}
$$

where we have set $\mathcal{C}\left(z, z^{\prime}\right) \equiv \mathcal{C}\left(z, z^{\prime} ; t, t\right)$ and $\mathcal{W}_{m}\left(z, z^{\prime}\right) \equiv$ $\mathcal{W}_{m}\left(z, z^{\prime} ; \tau=0\right)$. We note that $\Delta \mathcal{C}(0,0)$ is, in fact, the excess force variance.

The dimensionless integrals, $\mathcal{W}_{m}\left(z, z^{\prime}\right)$, are functions of just three dimensionless ratios: The ratio of the fluid viscosities, $\zeta / \eta$, which enters through $\chi$, defined in Eq. (35); the ratio of the plate separation to the propagative-diffusive boundary length scale $L / \delta$; and the ratio of the propagative-diffusive boundary length scale to the microscopic cutoff scale, $u_{\infty}=(\delta / a)^{2}$.

We tabulate our choices for these parameters, which correspond to a range of reasonable physical values, in Table [1. For the case of confined fluids, relevant in particular for our analysis, experiments and simulations on nano-slit confined water suggest that the bulk viscosity is recovered at boundary surface separations larger than approximately one nanometer [51 53]. To be on the safe side, we therefore take one nanometer as the implied microscopic cutoff $a$, but also indicate in Fig. 1(c) how the equal-time force correlators depend on this cutoff through the dimensionless parameter $u_{\infty}$.

We evaluate the frequency integrals $\mathcal{W}_{m}\left(z, z^{\prime}\right)$ numerically and plot the excess force variance, $\Delta \mathcal{C}(0,0)$, as a function of $L / \delta$ for $\zeta / \eta=1,3,5$ and 10 in Figs. 11(a) and 1(b) (solid curves). The cross-plate correlator, $\mathcal{C}(0, L)$, is shown by dashed curves in Fig. 1(b), where, for the sake of comparison, the curves for $\Delta \mathcal{C}(0,0)$ are replotted. In the figures, we plot the force correlators in units of $\left(k_{\mathrm{B}} T / \pi\right) \cdot\left(\rho_{0} c_{0}^{2} A\right)$.

Figs.1(a) and 1(b) show that both $\Delta \mathcal{C}(0,0)$ and $\mathcal{C}(0, L)$ become negative at small separations, $L / \delta \ll 1$, and

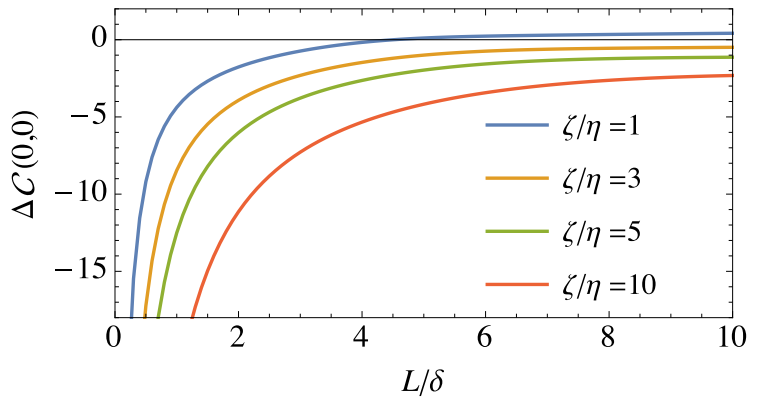

(a)

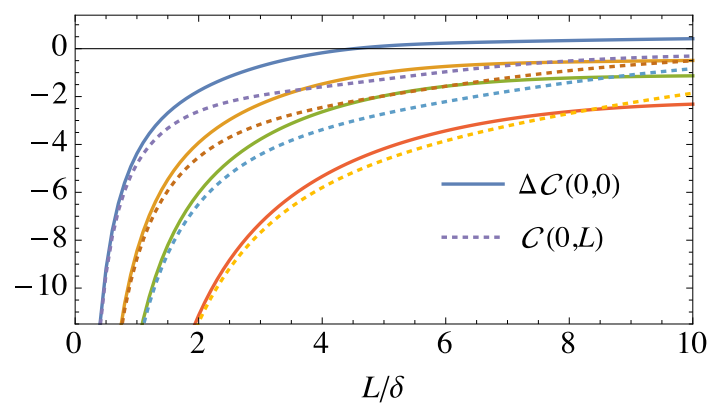

(b)

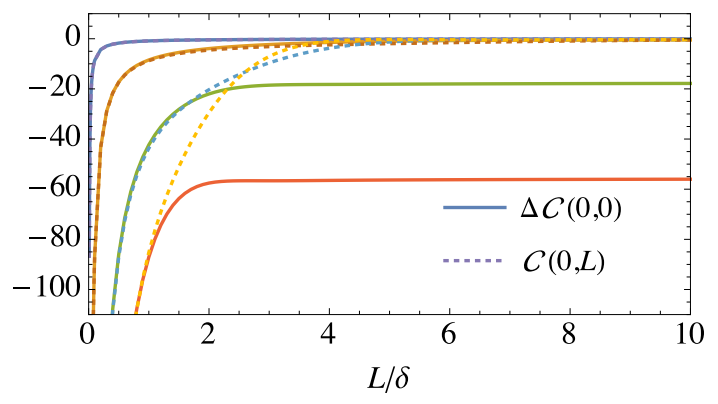

(c)

FIG. 1. (Color online) (a) Equal-time, excess same-plate force correlator (or force variance), $\Delta \mathcal{C}(0,0)$, as defined in Eq. (75), plotted as a function of $L / \delta$ for fixed $u_{\infty}=(\delta / a)^{2}=1$ and $\zeta / \eta=1,3,5$ and 10 (top to bottom). (b) Same as (a) but here we plot the equal-time, cross-plate force correlator $\mathcal{C}(0, L)$ as defined in Eq. (76) (dashed curves) and compare it with the equal-time, excess same-plate force correlator (solid curves). (c) Comparison of equal-time, excess same-plate force correlator, $\Delta \mathcal{C}(0,0)$ (solid curves), and equal-time, cross-plate force correlator, $\mathcal{C}(0, L)$ (dashed curves), plotted as a function of $L / \delta$ for fixed $\zeta / \eta=3$ and $u_{\infty}=0.1,1.0,5.0$ and 10.0 (top to bottom). We plot the force correlators in units of $\left(k_{\mathrm{B}} T / \pi\right) \cdot\left(\rho_{0} c_{0}^{2} A\right)$.

eventually diverge when $L / \delta \rightarrow 0$. In this limit, the curves for both these correlators overlap and, thus, they are approximated by the same limiting form. At large separations, $L / \delta \gg 1$, the cross-plate correlator tends to zero while the excess same-plate correlator tends to a constant depending on the viscosity parameters. Therefore, the cross-plate correlator remains negative over the whole range of separations, indicating that the two bounding surfaces are subjected to counter-phase correlations. The excess same-plate correlator, on the other hand, can be negative (for intermediate to large values of $\zeta / \eta$ ) or positive (for sufficiently small $\zeta / \eta$ ). Thus, when the two cor- 


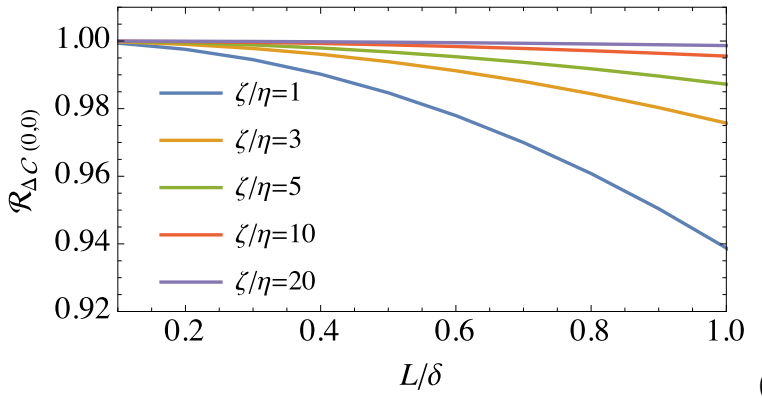

(a)

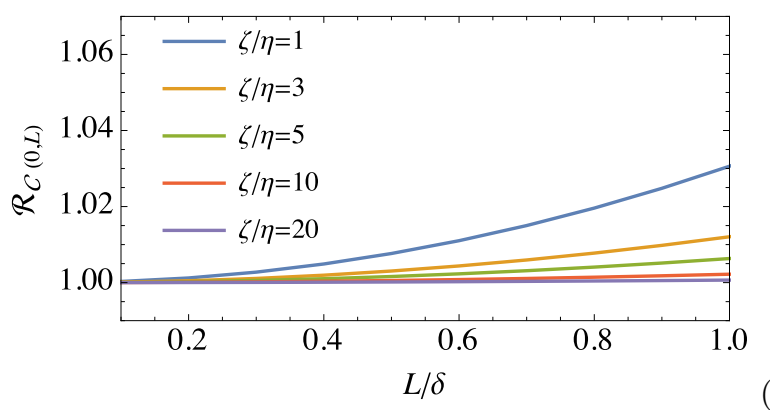

(b)

FIG. 2. (Color online) (a) Ratio of full numerical results of $\Delta \mathcal{C}(0,0)$ to the analytic limiting behavior of Eq. (77), shown on the graph by $\mathcal{R}_{\Delta C(0,0)}$, as a function of $L / \delta$ for $L / \delta \leq 1$ and $\zeta / \eta=1,3,5,10$ and 20. We fix $u_{\infty}=(\delta / a)^{2}=1$. (b) Same as (a) but for $\mathcal{C}(0, L)$.

relators are compared, as in Fig. 1(b), one can see that, at small to intermediate separations, the cross-plate correlator (dashed curves) is larger in magnitude than the excess same-plate correlator (solid curves); while, at large separations, it can become smaller than the latter. The difference between these two quantities decreases with increasing $\zeta / \eta$.

In addition, Fig. 1(c) demonstrates that the two correlators overlap for the whole range of plate separations for small $u_{\infty}$, illustrated by the overlap of the solid and dashed blue curves at $u_{\infty}=0.1$. For large $u_{\infty}$, such as at $u_{\infty}=10.0$, indicated by the solid red and dashed yellow curves, these correlators deviate significantly. At large plate separations, the same-plate correlator tends to a value that is independent of the plate separation, in agreement with the analytic result of Eq. (78), while the cross-plate correlator becomes independent of $u_{\infty}$ and decays with the separation, in agreement with Eq. (79).

\section{A. Analytic limits}

Beyond these numerical results, we can analytically calculate the small and large plate-separation limits and the limits of vanishing and infinite speed of sound (Burger's and incompressible limits, respectively). To study the small and large plate-separation cases, we first note that the frequency integrals of Eqs. (67) and (68) depend on $L / \delta$ through $\ell_{+}$and $\ell_{-}$, which are both linear in this ratio (see Eqs. (53) and (54)).

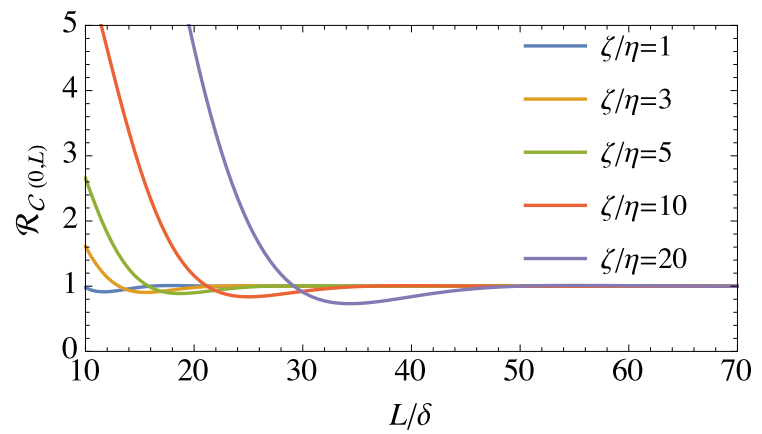

FIG. 3. (Color online) Ratio of full numerical results of $\mathcal{C}(0, L)$ to the analytic limiting behavior of Eq. (79), shown on the graph by $\mathcal{R}_{C(0, L)}$, as a function of $L / \delta$ for $L / \delta \gg 1$ and $\zeta / \eta=1,3,5,10$ and 20. We fix $u_{\infty}=(\delta / a)^{2}=1$.

Thus, in the small separation limit, $L / \delta \ll 1$, we can expand the frequency integrands as Taylor series in $L / \delta$ for both $\mathcal{W}_{m}(0,0)$ and $\mathcal{W}_{m}(0, L)$ and keep terms up to linear order in $L / \delta$. The resulting integrals are trivial, giving

$$
\Delta \mathcal{C}(0,0) \simeq \mathcal{C}(0, L) \simeq-\frac{k_{\mathrm{B}} T}{\pi} \frac{2(4 \eta / 3+\zeta) \eta A}{\rho_{0} a^{2} L} .
$$

These expressions agree with the full numerical results for $L / \delta \ll 1$, as we illustrate in Fig. 2. This figure shows the ratio of the full numerical result to the analytic approximation of Eq. (77) for $\Delta \mathcal{C}(0,0)$ (panel a) and $\mathcal{C}(0, L)$ (panel b). The plots show that the ratio in both cases tends to unity as $L / \delta$ becomes sufficiently small, but the domain of validity of the analytic approximation depends strongly on the ratio $\zeta / \eta$ and increases with increasing $\zeta / \eta$.

In the large separation limit, $L / \delta \gg 1$, the force variance reduces to the semi-infinite fluid result (see Appendix E],

$$
\begin{aligned}
& \Delta \mathcal{C}(0,0) \stackrel{L / \delta \rightarrow \infty}{=} \frac{k_{\mathrm{B}} T}{\pi} \frac{2 \rho_{0}^{2} c_{0}^{3} A}{\eta \chi}\left[\frac{2 \sqrt{z_{\infty}-1}}{z_{\infty}}\right. \\
& \left.\quad+\frac{8 \sqrt{2}}{3} \frac{z_{\infty}\left(3-z_{\infty}\right)}{\sqrt{z_{\infty}-1}} \sin ^{4}\left(\frac{1}{2} \arctan \left(z_{\infty}^{2}-1\right)\right)\right]
\end{aligned}
$$

where $z_{\infty}=\sqrt{1+x_{\infty}^{2}}$ and $x_{\infty}=\chi u_{\infty}=\chi \eta^{2} /\left(a^{2} \rho_{0}^{2} c_{0}^{2}\right)$.

The corresponding equal-time, cross-plate correlator tends to zero in the large plate-separation limit as

$$
\mathcal{C}(0, L) \simeq-\frac{k_{\mathrm{B}} T}{\pi} \frac{\pi \rho_{0} c_{0}^{2} A}{L} .
$$

This limiting behavior is independent of the ratio $\zeta / \eta$, as is clearly demonstrated by the plots of the ratio of the full numerical result to the analytic approximation of Eq. (79) in Fig. 3. However, the exact value of $L / \delta$ beyond which Eq. (79) is a reasonable approximation does depend on $\zeta / \eta$. 
In the incompressible fluid limit, we consider the leading contributions for $c_{0} \rightarrow \infty$, giving

$$
\Delta \mathcal{C}(0,0) \stackrel{c_{0} \rightarrow \infty}{=} \mathcal{C}(0, L) \stackrel{c_{0} \rightarrow \infty}{=}-\frac{k_{\mathrm{B}} T}{\pi} \frac{2(4 \eta / 3+\zeta) \eta A}{\rho_{0} a^{2} L} .
$$

There is a correspondence between the incompressible fluid limit and the small plate-separation limiting result of Eq. (77): At small separations, the fluid behaves as if it were incompressible.

In the limit of vanishing adiabatic speed of sound ("Burger's limit"), $c_{0} \rightarrow 0$, on the other hand, both $C(0,0)$ and $C(0, L)$ tend to zero as $c_{0}^{2}$.

\section{RESULTS FOR TIME-DEPENDENT CORRELATORS}

We now turn to the two-point, time-dependent correlators of the normal forces acting on the walls, which we compute numerically using Eqs. (72) and (73) for the same-plate and the cross-plate correlators, $\mathcal{C}\left(0,0 ; t, t^{\prime}\right)$ and $\mathcal{C}\left(0, L ; t, t^{\prime}\right)$, respectively.

We plot the behavior of the excess force correlator, Eq. (74), as a function of the rescaled time difference, $\tau=\left(t-t^{\prime}\right) / t_{0}$, for rescaled inter-plate separations $L / \delta=$ 1, 4 and 10 in Fig. 4(a). In Fig. 4(b), we show the timedependent behavior of the same quantity for $\zeta / \eta=1,3,5$ and 10 .

As seen in these figures, $\Delta \mathcal{C}\left(0,0 ; t, t^{\prime}\right)$ exhibits a damped oscillatory behavior in $\tau$. For $L / \delta \lesssim 3$, these oscillations are well described by a function of the form $\alpha \sin \left(u_{\infty} \tau\right) / \tau$, where $\alpha$ is a function of the viscosity ratio, $\zeta / \eta$, the dimensionless cutoff, $u_{\infty}$, and the rescaled plate separation, $L / \delta$. For $L / \delta \gtrsim 3$, this simple behavior breaks down, although $\Delta \mathcal{C}\left(0,0 ; t, t^{\prime}\right)$ remains oscillatory with an amplitude that gradually decreases for large $\tau$. For the example of water at room temperature, with the plate separation $L / \delta=1, \zeta / \eta=3$ and cutoff $u_{\infty}=1$, we find $\alpha=-8.5(3)$.

The cross-plate force correlator shows a similar timedependent behavior as the excess same-plate correlator, and the onset of irregular oscillations occurs for similar values of $L / \delta$. We compare the same-plate (solid curves) and cross-plate (dashed curves) correlators in Fig. 5 .

We plot the difference between the excess same-plate correlator and the cross-plate correlator, defined as $\delta \mathcal{C} \equiv$ $\Delta \mathcal{C}\left(0,0 ; t, t^{\prime}\right)-\mathcal{C}\left(0, L ; t, t^{\prime}\right)$, in Fig. 6. This plot shows that the two correlators exhibit similar period of oscillations for a wide range of viscosities, especially at small to intermediate inter-plate separations. In the special case of equal-time correlators with $\tau=0$, one can see a nonmonotonic behavior for the two correlators in Fig. 6(a): At small inter-plate separations, $\left.\delta \mathcal{C}\right|_{\tau=0}$ is positive and increases by increasing $L / \delta$, but this trend changes at around $L / \delta \simeq 3$, and then tends to zero for large $L / \delta$.

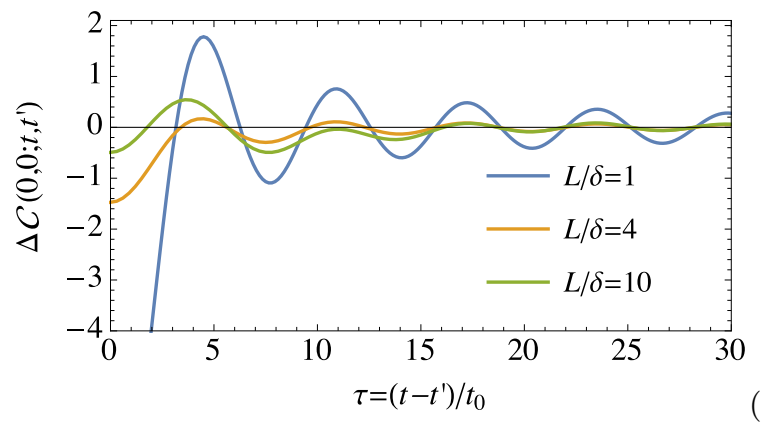

(a)

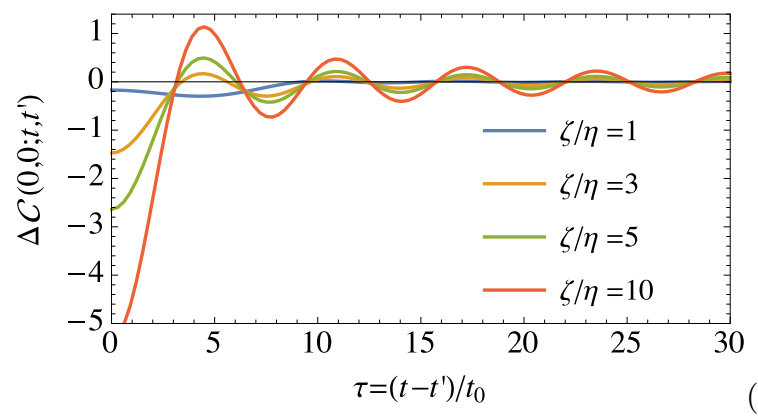

(b)

FIG. 4. (Color online) (a) Time-dependent, excess same-plate force correlator, $\Delta \mathcal{C}\left(0,0 ; t, t^{\prime}\right)$, as defined in Eq. (72), plotted as a function of the rescaled time difference, $\tau=\left(t-t^{\prime}\right) / t_{0}$, for fixed $u_{\infty}=(\delta / a)^{2}=1, \zeta / \eta=3$ and $L / \delta=1,4$ and 10 , as indicated on the graph. (b) Same as (a) but here we show the results for fixed $u_{\infty}=(\delta / a)^{2}=1, L / \delta=4$ and $\zeta / \eta=1,3,5$ and 10 .

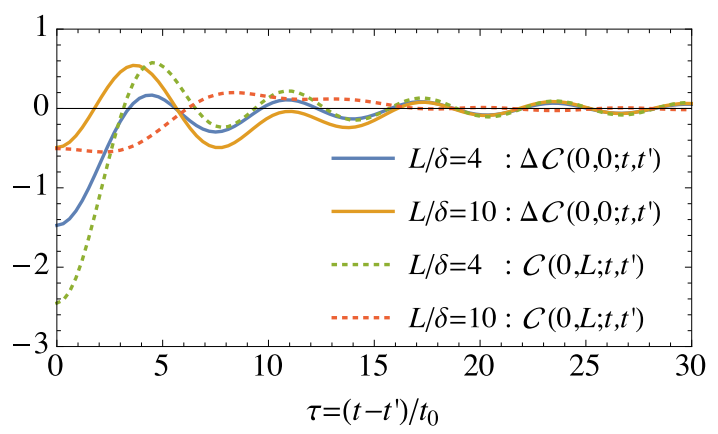

FIG. 5. (Color online) Time-dependent, excess sameplate force correlator, $\Delta \mathcal{C}\left(0,0 ; t, t^{\prime}\right)$ (solid curves), compared with the time-dependent cross-plate correlator, $\mathcal{C}\left(0, L ; t, t^{\prime}\right)$ (dashed curves), for fixed $u_{\infty}=(\delta / a)^{2}=1, \zeta / \eta=3$ and at two different rescaled inter-plate separations, $L / \delta=4$ and 10 , as indicated on the graph.

\section{CONCLUSION AND DISCUSSION}

We have revisited the problem of long-range, fluctuation-induced (or Casimir-like) hydrodynamic interactions within the context of Landau-Lifshitz's linear, stochastic hydrodynamics in a classical, compressible, viscous fluid confined between two rigid, planar walls with no-slip boundary conditions and in the absence of heat transfer. We show conclusively that, at 


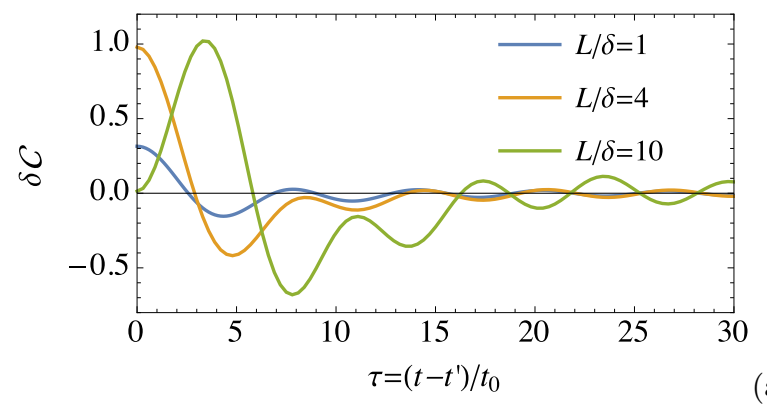

(a)

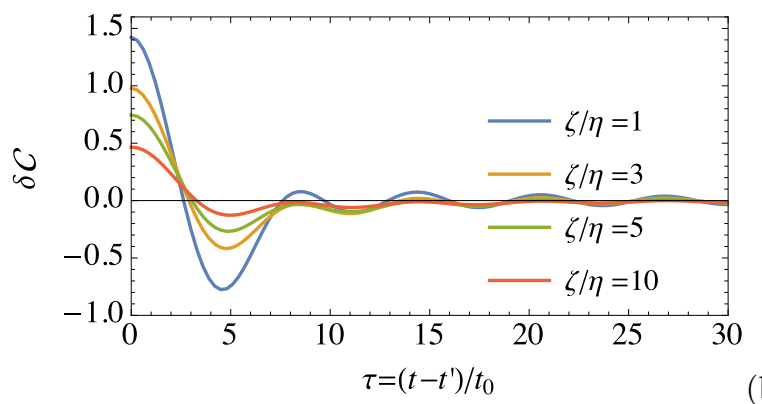

(b)

FIG. 6. (Color online) (a) The difference between the excess same-plate and the cross-plate force correlators defined as $\delta \mathcal{C} \equiv \Delta \mathcal{C}\left(0,0 ; t, t^{\prime}\right)-\mathcal{C}\left(0, L ; t, t^{\prime}\right)$, plotted as a function of the rescaled time difference, $\tau=\left(t-t^{\prime}\right) / t_{0}$, for fixed $u_{\infty}=(\delta / a)^{2}=1$ and $\zeta / \eta=3$, and $L / \delta=1,4$ and 10 . (b) Fixed $L / \delta=4$ and $\zeta / \eta=1,3,5$ and 10 as indicated on the graph.

this level and within the pertinent approximations, there is no standard or primary Casimir effect manifest in the average value of the interaction force between the fluid boundaries. Nevertheless, we show that there does exist a secondary Casimir effect in the variance of the normal force as well as in the cross-correlation function of the normal force between the bounding surfaces. Fluctuations in such effective fluctuation-induced forces have been investigated in other Casimir-like contexts [22, 42] and in disordered charged systems [43 45].

We derive general expressions for the two-point, timedependent, force correlations and, thus, show that:

1. The variance of the fluctuation-induced force is finite and independent of the separation between the bounding surfaces for large separations;

2. The equal-time, cross-plate force correlation exhibits a long-range decay with the inverse plate separation that is independent of the fluid viscosities;

3. The time-dependent force correlations exhibit a damped oscillatory behavior for small and intermediate inter-plate separations that grows more irregular at large separations.

Our calculation is based on the Landau-Lifshitz linear stochastic hydrodynamics and, therefore, does not include putative non-linear effects [37]. If such effects could be brought into the fold, they would have to be considered consistently for all variables. Moreover, we find that incorporating compressibility does not completely obliterate all fluctuation effects, contrary to previous attempts, based on contour integration in the complex plane, that required the limiting behavior of hydrodynamics at infinite frequencies [38]. In fact, our calculation explicitly includes the scale at which the macroscopic hydrodynamics breaks down. The limit of vanishing compressibility is non-trivial and has to be taken carefully, because it can never be derived from a realistic inter-particle potential with infinite stiffness [39].

We interpret the non-zero hydrodynamic force correlations predicted in this work as a modification of the thermal stochastic force correlations that act on a Brownian particle in a fluid. Since the force correlator depends on the separation between the particles, the bathmediated force fluctuations between the particles would modify the particles' Langevin dynamics and thus, in principle, should be detectable [54, 55]. The separation dependence of the normal force cross-correlation function represents an interesting case of colloidal bodies which do not interact directly, but are driven by correlated noise sources that can provide an alternative mechanism which can produce non-trivial, ordered steady states [56]. We have considered infinite bounding surfaces, so our results are not strictly applicable to the case of finite particles, but our calculation can be straightforwardly generalized to include a spherical geometry, which would also admit an analytic, albeit much more complicated, solution. Moreover, we intend to include the effects of heat transfer in a future calculation.

For experimental verification of our results, we again note that one would have to generalize our calculation to the case of two spheres in a fluctuating hydrodynamic medium. This is different from the existing analysis of fluctuations of two unconnected, but hydrodynamically interacting spheres 55], a problem in some sense dual to ours. In order to exploit this connection, our first step will be to calculate the cross-correlation function for two spherical particles.

\section{ACKNOWLEDGMENTS}

This work has been partially funded by the U.S. Department of Energy. C.M. was supported in part by the U.S. National Science Foundation under Grant NSF PHY10-034278. A.N. acknowledges partial support from the Royal Society, the Royal Academy of Engineering, and the British Academy. B.-S.L. and R.P. also acknowledge the financial support of the Agency for research and development of Slovenia (ARRS) under the bilateral SLO-A Grant No. N1-0019. We acknowledge illuminating discussions with M. Kardar in the KITP program on The Theory and Practice of Fluctuation-Induced Interactions (2008). R.P. would like to thank Joel Cohen for his careful reading of the manuscript and for his comments. 


\section{Appendix A: Derivation of the force correlator,} Eq. (34)

In this Appendix, we derive the explicit expression, Eq. (34), for the force correlator defined in terms of the stress tensor in Eq. (29). Our starting point is the general expression

$$
\left\langle\mathcal{F}_{i}^{(1)}(t) \mathcal{F}_{j}^{(1)}(t)\right\rangle=\iint_{\Gamma}\left\langle\sigma_{i k}^{(1)}(\mathbf{r} ; t) \sigma_{j l}^{(1)}\left(\mathbf{r}^{\prime} ; t\right)\right\rangle \mathrm{d} A_{k} \mathrm{~d} A_{l}^{\prime},
$$

where repeated subindices are summed over. In principle, this equation represents nine components of the force variance, each of which has nine contributions. For this work, we are interested in only the $i=j=z$ component of the force acting on the plane parallel to the boundaries. We thus have

$$
\begin{aligned}
\mathcal{C}\left(z, z^{\prime} ; t, t^{\prime}\right)=\left\langle\mathcal{F}_{z}^{(1)}(z ; t) \mathcal{F}_{z}^{(1)}\left(z^{\prime} ; t^{\prime}\right)\right\rangle \\
\quad=\iint_{A}\left\langle\sigma_{z z}^{(1)}(\mathbf{r} ; t) \sigma_{z z}^{(1)}\left(\mathbf{r}^{\prime} ; t^{\prime}\right)\right\rangle \mathrm{d} A_{z} \mathrm{~d} A_{z}^{\prime},
\end{aligned}
$$

where $A$ is the surface area for each of the plates and $\mathrm{d} A_{z}=\mathrm{d} x \mathrm{~d} y$ and $\mathrm{d} A_{z}^{\prime}=\mathrm{d} x^{\prime} \mathrm{d} y^{\prime}$. The first-order stress tensor is given by

$$
\begin{aligned}
& \sigma_{i j}^{(1)}(\mathbf{r} ; t)=\eta\left(\nabla_{i} v_{j}^{(1)}(\mathbf{r} ; t)+\nabla_{j} v_{i}^{(1)}(\mathbf{r} ; t)\right) \\
& -\left[\left(\frac{2 \eta}{3}-\zeta\right) \nabla_{k} v_{k}^{(1)}(\mathbf{r} ; t)+c_{0}^{2} \rho^{(1)}(\mathbf{r} ; t)\right] \delta_{i j}+S_{i j}(\mathbf{r} ; t) .
\end{aligned}
$$

In calculating the force correlator, which follows by inserting (A33) into (A2), we realize that we are ultimately interested in these correlation functions evaluated at the boundaries with no-slip boundary conditions. Therefore, the terms that contain a derivative with respect to the transverse directions acting on the velocity field will vanish. On the other hand, the spatial (surface) integral over the transverse correlation function $c_{z z}^{\mathrm{TT}}\left(\mathbf{r}, \mathbf{r}^{\prime} ; t, t^{\prime}\right)=$ $\left\langle v_{z}^{\mathrm{T}}(\mathbf{r} ; t) v_{z}^{\mathrm{T}}\left(\mathbf{r}^{\prime} ; t^{\prime}\right)\right\rangle$ also vanishes (see Appendix $\left.\mathbf{B}\right)$. It is also straightforward to show that the terms containing cross correlations between the random stress tensor and other fluctuating fields vanish; this is because these terms turn out to be proportional to $\nabla_{z} \delta\left(z-z^{\prime}\right)$, which is zero for $z \neq z^{\prime}$ and can also be set to zero for $z=z^{\prime}$ by using a standard regularization scheme (e.g., by considering the Dirac delta function as a limiting form of a Gaussian function). Hence, the expression for the force correlator is:

$$
\begin{aligned}
\mathcal{C}\left(z, z^{\prime} ; t,\right. & \left.t^{\prime}\right)=\iint_{A} \mathrm{~d} A_{z} \mathrm{~d} A_{z}^{\prime}\left\{\left\langle S_{z z}(\mathbf{r} ; t) S_{z z}\left(\mathbf{r}^{\prime} ; t^{\prime}\right)\right\rangle\right. \\
+ & \left(\frac{4 \eta}{3}+\zeta\right)^{2} \nabla_{z} \nabla_{z}^{\prime}\left\langle v_{z}^{(1)}(\mathbf{r} ; t) v_{z}^{(1)}\left(\mathbf{r}^{\prime} ; t^{\prime}\right)\right\rangle \\
- & \left(\frac{4 \eta}{3}+\zeta\right) c_{0}^{2} \nabla_{z}\left\langle v_{z}^{(1)}(\mathbf{r} ; t) \rho^{(1)}\left(\mathbf{r}^{\prime} ; t^{\prime}\right)\right\rangle \\
- & \left(\frac{4 \eta}{3}+\zeta\right) c_{0}^{2} \nabla_{z}^{\prime}\left\langle\rho^{(1)}(\mathbf{r} ; t) v_{z}^{(1)}\left(\mathbf{r}^{\prime} ; t^{\prime}\right)\right\rangle \\
& \left.+c_{0}^{4}\left\langle\rho^{(1)}(\mathbf{r} ; t) \rho^{(1)}\left(\mathbf{r}^{\prime} ; t^{\prime}\right)\right\rangle\right\} .
\end{aligned}
$$

Finally, the contributions from the correlation functions between the density and velocity fields (third and fourth terms in Eq. (A4) ) cancel out (see App. D). Therefore, we find

$$
\begin{aligned}
& \mathcal{C}\left(z, z^{\prime} ; t, t^{\prime}\right)= \mathcal{P}_{0}\left(z, z^{\prime} ; t, t^{\prime}\right) \\
&+\iint_{A} \mathrm{~d} A_{z} \mathrm{~d} A_{z}^{\prime}\left\{c_{0}^{4} c^{\rho \rho}\left(\mathbf{r}, \mathbf{r}^{\prime} ; t, t^{\prime}\right)\right. \\
&\left.\quad+\eta^{2} \chi^{2} \nabla_{z} \nabla_{z}^{\prime} c_{z z}^{\mathrm{LL}}\left(\mathbf{r}, \mathbf{r}^{\prime} ; t, t^{\prime}\right)\right\},
\end{aligned}
$$

where $\mathcal{P}_{0}\left(z, z^{\prime} ; t, t^{\prime}\right)=2 k_{\mathrm{B}} T \eta \chi A \delta\left(z-z^{\prime}\right) \delta\left(t-t^{\prime}\right)$ and $\chi=$ $(4 / 3+\zeta / \eta)$. This is nothing but Eq. (34).

\section{Appendix B: Transverse velocity correlator does not} contribute

The derivation of the correlation function for the transverse velocity fields largely follows that for the longitudinal components (Sec. IVC). In terms of the stochastic stress, the transverse velocity correlation function is

$$
\begin{aligned}
\left\langle v_{i}^{\mathrm{T}}(\mathbf{r} ; t) v_{j}^{\mathrm{T}}\left(\mathbf{r}^{\prime} ; t^{\prime}\right)\right\rangle=\int \mathrm{d} t^{\prime \prime} \int \mathrm{d} t^{\prime \prime \prime} \int \mathrm{d}^{3} \mathbf{r}^{\prime \prime} \int \mathrm{d}^{3} \mathbf{r}^{\prime \prime \prime} \\
\times G^{\mathrm{T}}\left(\mathbf{r}, \mathbf{r}^{\prime \prime} ; t-t^{\prime \prime}\right) G^{\mathrm{T}}\left(\mathbf{r}^{\prime}, \mathbf{r}^{\prime \prime \prime} ; t^{\prime}-t^{\prime \prime \prime}\right) \\
\quad \times\left\langle\Sigma_{i}^{\mathrm{T}}\left(\mathbf{r}^{\prime \prime} ; t^{\prime \prime}\right) \Sigma_{j}^{\mathrm{T}}\left(\mathbf{r}^{\prime \prime \prime} ; t^{\prime \prime \prime}\right)\right\rangle .
\end{aligned}
$$

Here, the transverse Green function satisfies

$$
\left[\nabla_{z}^{2}-q^{2}\right] \widetilde{G}^{\mathrm{T}}\left(z, z^{\prime \prime} ; \mathbf{k} ; \omega\right)=\frac{1}{\eta} \delta\left(z-z^{\prime \prime}\right),
$$

where $q^{2}=\left(k^{2}-i \omega \rho_{0} / \eta\right)$. The solution for parallel-plane boundaries is

$$
\widetilde{G}^{\mathrm{T}}\left(z, z^{\prime \prime} ; \mathbf{k} ; \omega\right)=g_{1}^{\mathrm{T}} e^{-q z}+g_{2}^{\mathrm{T}} e^{q(z-L)}-\frac{1}{2 \eta q} e^{-q\left|z-z^{\prime \prime}\right|},
$$

with constants of integration given by

$$
\begin{aligned}
g_{1}^{\mathrm{T}} & =\frac{1}{2 q \eta} \operatorname{csch}(q L) \sinh \left(q\left(L-z^{\prime \prime}\right)\right), \\
g_{2}^{\mathrm{T}} & =\frac{1}{2 q \eta} \operatorname{csch}(q L) \sinh \left(q z^{\prime \prime}\right) .
\end{aligned}
$$


Recalling the stochastic properties of the stress tensor, which are

$$
\begin{gathered}
\left\langle\Sigma_{i}^{\mathrm{T}}(\mathbf{r} ; t) \Sigma_{j}^{\mathrm{T}}\left(\mathbf{r}^{\prime} ; t^{\prime}\right)\right\rangle=2 k_{\mathrm{B}} T \eta\left(\nabla_{k} \nabla_{k}^{\prime} \delta_{i j}-\nabla_{i} \nabla_{j}^{\prime}\right) \\
\times \delta\left(\mathbf{r}-\mathbf{r}^{\prime}\right) \delta\left(t-t^{\prime}\right)
\end{gathered}
$$

in the time domain, we can immediately carry out one of the time integrals and one of the the spatial integrals. Moreover, we are only concerned with the $i=j=z$ component, which leads to

$$
\begin{aligned}
& c_{z z}^{\mathrm{TT}}\left(\mathbf{r}, \mathbf{r}^{\prime} ; t, t^{\prime}\right)=2 k_{\mathrm{B}} T \eta \int \mathrm{d} t^{\prime \prime} \int \mathrm{d}^{3} \mathbf{r}^{\prime \prime} \\
& \times\left\{\nabla_{k}^{\prime \prime} G^{\mathrm{T}}\left(\mathbf{r}, \mathbf{r}^{\prime \prime} ; t-t^{\prime \prime}\right) \nabla_{k}^{\prime \prime} G^{\mathrm{T}}\left(\mathbf{r}^{\prime}, \mathbf{r}^{\prime \prime} ; t^{\prime}-t^{\prime \prime}\right)\right. \\
& \left.\quad-\nabla_{z}^{\prime \prime} G^{\mathrm{T}}\left(\mathbf{r}, \mathbf{r}^{\prime \prime} ; t-t^{\prime \prime}\right) \nabla_{z}^{\prime \prime} G^{\mathrm{T}}\left(\mathbf{r}^{\prime}, \mathbf{r}^{\prime \prime} ; t^{\prime}-t^{\prime \prime}\right)\right\} .
\end{aligned}
$$

Integrating by parts, this becomes

$$
\begin{aligned}
& c_{z z}^{\mathrm{TT}}\left(\mathbf{r}, \mathbf{r}^{\prime} ; t, t^{\prime}\right)=-2 k_{\mathrm{B}} T \eta \int \mathrm{d} t^{\prime \prime} \int \mathrm{d}^{3} \mathbf{r}^{\prime \prime} \\
& \times\left[\left(\nabla^{\prime \prime 2}-\nabla_{z}^{\prime \prime 2}\right) G^{\mathrm{T}}\left(\mathbf{r}, \mathbf{r}^{\prime \prime} ; t-t^{\prime \prime}\right)\right] G^{\mathrm{T}}\left(\mathbf{r}^{\prime}, \mathbf{r}^{\prime \prime} ; t^{\prime}-t^{\prime \prime}\right) .
\end{aligned}
$$

Moving to the frequency representation and substituting the translation-invariant form of the Green function, analogous to Eq. (39), we obtain

$$
\begin{gathered}
c_{z z}^{\mathrm{TT}}\left(\mathbf{r}, \mathbf{r}^{\prime} ; t, t^{\prime}\right)=2 k_{\mathrm{B}} T \eta \int \frac{\mathrm{d} \omega^{\prime}}{2 \pi} e^{i \omega^{\prime}\left(t-t^{\prime}\right)} \int \mathrm{d} z^{\prime \prime} \int \frac{\mathrm{d}^{2} \mathbf{k}}{(2 \pi)^{2}} \\
\times e^{i \mathbf{k} \cdot\left(\mathbf{s}-\mathbf{s}^{\prime}\right)} \mathbf{k}^{2} \widetilde{G}^{\mathrm{T}}\left(z, z^{\prime \prime} ; \mathbf{k} ;-\omega^{\prime}\right) \widetilde{G}^{\mathrm{T}}\left(z^{\prime}, z^{\prime \prime} ;-\mathbf{k} ; \omega^{\prime}\right) .
\end{gathered}
$$

Here, we have integrated over $x^{\prime \prime}$ and $y^{\prime \prime}$, which generates a wavenumber delta function $\delta\left(\mathbf{k}+\mathbf{k}^{\prime}\right)$ that simplifies one of the wavenumber integrals.

We calculate the force variance, $\mathcal{C}\left(z, z^{\prime} ; t, t^{\prime}\right)$, by integrating the velocity correlation function over the boundaries of the fluid, i.e. over $x, x^{\prime}, y$, and $y^{\prime}$ (see Eq. (34)). The only dependence on these variables occurs in the exponential function $e^{i \mathbf{k} \cdot\left(\mathbf{s}-\mathbf{s}^{\prime}\right)}$. Thus, this integral generates a second wavenumber Dirac delta function, $\delta(\mathbf{k})$. It is now straightforward to see that the wavenumber integral vanishes: The product of the Green functions at $\mathbf{k}=\mathbf{0}$ is finite and consequently the factor of $\mathbf{k}^{2}$ ensures that the integral vanishes.

\section{Appendix C: Derivation of the density correlator}

Here, we calculate the correlation function of the density fields, $c^{\rho \rho}$, given in Eq. (62). We start with the continuity equation, Eq. (11), which can be written as

$$
\dot{\rho}^{(1)}(\mathbf{r} ; t)+\rho_{0} \nabla \cdot \mathbf{v}^{\mathrm{L}}(\mathbf{r} ; t)=0,
$$

where the dot indicates a time derivative. From here we construct the correlation function

$$
\left\langle\dot{\rho}^{(1)}(\mathbf{r} ; t) \dot{\rho}^{(1)}\left(\mathbf{r}^{\prime} ; t^{\prime}\right)\right\rangle=\rho_{0}^{2} \nabla_{i} \nabla^{\prime}{ }_{j}\left\langle v_{i}^{\mathrm{L}}(\mathbf{r} ; t) v_{j}^{\mathrm{L}}\left(\mathbf{r}^{\prime} ; t^{\prime}\right)\right\rangle .
$$

By introducing Fourier components, we can cast the left-hand side of this equation into the form

$$
\begin{aligned}
\left\langle\dot{\rho}^{(1)}(\mathbf{r} ; t) \dot{\rho}^{(1)}\left(\mathbf{r}^{\prime} ; t^{\prime}\right)\right\rangle= & \int \frac{\mathrm{d} \omega}{2 \pi} \int \frac{\mathrm{d} \omega^{\prime}}{2 \pi} e^{-i\left(\omega t+\omega^{\prime} t^{\prime}\right)} \\
& \times\left\langle\widetilde{\rho}^{(1)}(\mathbf{r} ; \omega) \widetilde{\rho}^{(1)}\left(\mathbf{r}^{\prime} ; \omega^{\prime}\right)\right\rangle .
\end{aligned}
$$

The correlation function of the right-hand side of Eq. (C2) is given by

$$
\begin{aligned}
& c_{i j}^{\mathrm{LL}}\left(\mathbf{r}, \mathbf{r}^{\prime} ; t, t^{\prime}\right)=2 k_{\mathrm{B}} T \eta \chi \int \frac{\mathrm{d} \omega}{2 \pi} \int \mathrm{d} \omega^{\prime} e^{-i\left(\omega t+\omega^{\prime} t^{\prime}\right)} \quad(\mathrm{C} 4) \\
& \quad \times \delta\left(\omega+\omega^{\prime}\right) \int \mathrm{d}^{3} \mathbf{r}^{\prime \prime} \nabla_{i} \widetilde{G}^{\mathrm{L}}\left(\mathbf{r}, \mathbf{r}^{\prime \prime} ; \omega\right) \nabla_{j}^{\prime} \widetilde{G}^{\mathrm{L}}\left(\mathbf{r}^{\prime}, \mathbf{r}^{\prime \prime} ; \omega^{\prime}\right) .
\end{aligned}
$$

Combining Eqs. (C2), (C3) and (C4), we write

$$
\begin{aligned}
& \left\langle\widetilde{\dot{\rho}}^{(1)}(\mathbf{r} ; \omega) \widetilde{\dot{\rho}}^{(1)}\left(\mathbf{r}^{\prime} ; \omega^{\prime}\right)\right\rangle=4 \pi k_{\mathrm{B}} T \rho_{0}^{2} \eta \chi \delta\left(\omega+\omega^{\prime}\right) \\
& \quad \times \int \mathrm{d}^{3} \mathbf{r}^{\prime \prime} \nabla_{i} \nabla_{i}^{\prime \prime} \widetilde{G}^{\mathrm{L}}\left(\mathbf{r}, \mathbf{r}^{\prime \prime} ; \omega\right) \nabla_{j}^{\prime} \nabla_{j}^{\prime \prime} \widetilde{G}^{\mathrm{L}}\left(\mathbf{r}^{\prime}, \mathbf{r}^{\prime \prime} ; \omega^{\prime}\right) .
\end{aligned}
$$

Then, substituting this result into Eq. (C3) gives

$$
\begin{aligned}
& \left\langle\dot{\rho}^{(1)}(\mathbf{r} ; t) \dot{\rho}^{(1)}\left(\mathbf{r}^{\prime} ; t\right)\right\rangle=4 \pi k_{\mathrm{B}} T \rho_{0}^{2} \eta \chi \int \mathrm{d} \omega^{\prime} e^{i \omega^{\prime}\left(t-t^{\prime}\right)} \\
& \quad \times \int \mathrm{d}^{3} \mathbf{r}^{\prime \prime} \nabla_{i} \nabla_{i}^{\prime \prime} \widetilde{G}^{\mathrm{L}}\left(\mathbf{r}, \mathbf{r}^{\prime \prime} ;-\omega^{\prime}\right) \nabla_{j}^{\prime} \nabla_{j}^{\prime \prime} \widetilde{G}^{\mathrm{L}}\left(\mathbf{r}^{\prime}, \mathbf{r}^{\prime \prime} ; \omega^{\prime}\right) .
\end{aligned}
$$

Several more steps are needed. First of all, we note that for the Fourier components of the density field, we have

$$
\begin{aligned}
& \left\langle\widetilde{\rho}^{(1)}(\mathbf{r} ; \omega) \widetilde{\rho}^{(1)}\left(\mathbf{r}^{\prime} ; \omega^{\prime}\right)\right\rangle=-4 \pi k_{\mathrm{B}} T \rho_{0}^{2} \eta \chi \frac{\delta\left(\omega+\omega^{\prime}\right)}{\omega \omega^{\prime}} \\
& \quad \times \int \mathrm{d}^{3} \mathbf{r}^{\prime \prime} \nabla_{i} \nabla_{i}^{\prime \prime} \widetilde{G}^{\mathrm{L}}\left(\mathbf{r}, \mathbf{r}^{\prime \prime} ; \omega\right) \nabla_{j}^{\prime} \nabla_{j}^{\prime \prime} \widetilde{G}^{\mathrm{L}}\left(\mathbf{r}^{\prime}, \mathbf{r}^{\prime \prime} ; \omega^{\prime}\right),
\end{aligned}
$$

and, therefore, we finally find

$$
\begin{aligned}
& c^{\rho \rho}\left(\mathbf{r}, \mathbf{r}^{\prime} ; t, t^{\prime}\right)=\frac{k_{\mathrm{B}} T}{\pi} \rho_{0}^{2} \eta \chi \int \frac{\mathrm{d} \omega^{\prime}}{\omega^{\prime 2}} e^{i \omega^{\prime}\left(t-t^{\prime}\right)} \\
& \times \int \mathrm{d}^{3} \mathbf{r}^{\prime \prime} \nabla_{i} \nabla_{i}^{\prime \prime} \widetilde{G}^{\mathrm{L}}\left(\mathbf{r}, \mathbf{r}^{\prime \prime} ;-\omega^{\prime}\right) \nabla_{j}^{\prime} \nabla_{j}^{\prime \prime} \widetilde{G}^{\mathrm{L}}\left(\mathbf{r}^{\prime}, \mathbf{r}^{\prime \prime} ; \omega^{\prime}\right) .
\end{aligned}
$$

Here, the relevant derivatives are given by

$$
\begin{aligned}
\nabla_{i} \nabla_{i}^{\prime \prime} \widetilde{G}^{\mathrm{L}}\left(\mathbf{r}, \mathbf{r}^{\prime \prime} ; \omega^{\prime}\right)=\int \frac{\mathrm{d}^{2} \mathbf{k}}{(2 \pi)^{2}} e^{i \mathbf{k} \cdot\left(\mathbf{s}-\mathbf{s}^{\prime \prime}\right)} \\
\quad \times\left(\mathbf{k}^{2}+\nabla_{z} \nabla_{z}^{\prime \prime}\right) \widetilde{G}^{\mathrm{L}}\left(z, z^{\prime \prime} ; \mathbf{k} ; \omega^{\prime}\right) .
\end{aligned}
$$

The force variance, $\mathcal{C}\left(z, z^{\prime} ; t, t^{\prime}\right)$, follows by integrating the velocity correlation function over the boundaries of the fluid, i.e. over $x, x^{\prime}, y$, and $y^{\prime}$ (see Eq. (34)). The only dependence on these variables occurs in the exponential function $e^{i \mathbf{k} \cdot\left(\mathbf{s}-\mathbf{s}^{\prime}\right)}$ and consequently this integral generates a Dirac delta function over the transverse wavenumbers, $(2 \pi)^{2} A \delta\left(\mathbf{k}+\mathbf{k}^{\prime}\right)$. This leads directly to Eq. (62). 
Appendix D: Density-velocity cross-correlator does not contribute

To calculate the density-velocity cross-correlator, $c_{i}^{\mathrm{L} \rho}\left(\mathbf{r}, \mathbf{r}^{\prime} ; t, t^{\prime}\right)$, we first construct the cross-correlation function

$$
\left\langle v_{i}^{\mathrm{L}}(\mathbf{r} ; t) \dot{\rho}^{(1)}\left(\mathbf{r}^{\prime}, t^{\prime}\right)\right\rangle=-\rho_{0} \nabla_{j}^{\prime}\left\langle v_{i}^{\mathrm{L}}(\mathbf{r} ; t) v_{j}^{\mathrm{L}}\left(\mathbf{r}^{\prime} ; t^{\prime}\right)\right\rangle .
$$

Following a similar line of reasoning to that for the density-density correlation function, we can write

$$
\begin{aligned}
& \left\langle v_{i}^{\mathrm{L}}(\mathbf{r} ; \omega) \widetilde{\rho}^{(1)}\left(\mathbf{r}^{\prime} ; \omega^{\prime}\right)\right\rangle=-4 \pi k_{\mathrm{B}} T \rho_{0} \eta \chi \delta\left(\omega+\omega^{\prime}\right) \\
& \times \int \mathrm{d}^{3} \mathbf{r}^{\prime \prime} \nabla_{i}^{\prime \prime} \widetilde{G}^{\mathrm{L}}\left(\mathbf{r}, \mathbf{r}^{\prime \prime} ; \omega\right) \nabla_{j}^{\prime} \nabla_{j}^{\prime \prime} \widetilde{G}^{\mathrm{L}}\left(\mathbf{r}^{\prime}, \mathbf{r}^{\prime \prime} ; \omega^{\prime}\right) .
\end{aligned}
$$

This equation leads to

$$
\begin{aligned}
& \left\langle v_{i}^{\mathrm{L}}(\mathbf{r} ; \omega) \widetilde{\rho}^{(1)}\left(\mathbf{r}^{\prime} ; \omega^{\prime}\right)\right\rangle=4 \pi i k_{\mathrm{B}} T \rho_{0} \eta \chi \frac{\delta\left(\omega+\omega^{\prime}\right)}{\omega^{\prime}} \\
& \times \int \mathrm{d}^{3} \mathbf{r}^{\prime \prime} \nabla_{i}^{\prime \prime} \widetilde{G}^{\mathrm{L}}\left(\mathbf{r}, \mathbf{r}^{\prime \prime} ; \omega\right) \nabla_{j}^{\prime} \nabla_{j}^{\prime \prime} \widetilde{G}^{\mathrm{L}}\left(\mathbf{r}^{\prime}, \mathbf{r}^{\prime \prime} ; \omega^{\prime}\right) .
\end{aligned}
$$

Thus, we obtain

$$
\begin{aligned}
& c_{i}^{\mathrm{L} \rho}\left(\mathbf{r}, \mathbf{r}^{\prime} ; t, t^{\prime}\right)=i \frac{k_{\mathrm{B}} T}{\pi} \rho_{0} \eta \chi \int \frac{\mathrm{d} \omega^{\prime}}{\omega^{\prime}} e^{i \omega^{\prime}\left(t-t^{\prime}\right)} \\
& \times \int \mathrm{d}^{3} \mathbf{r}^{\prime \prime} \nabla_{i}^{\prime \prime} \widetilde{G}^{\mathrm{L}}\left(\mathbf{r}, \mathbf{r}^{\prime \prime} ;-\omega^{\prime}\right) \nabla_{j}^{\prime} \nabla_{j}^{\prime \prime} \widetilde{G}^{\mathrm{L}}\left(\mathbf{r}^{\prime}, \mathbf{r}^{\prime \prime} ; \omega^{\prime}\right) .
\end{aligned}
$$

We now introduce the translational invariant form of the Green function and take $i=z$. The double spatial integral generates a double wavenumber Dirac delta function, giving

$$
\begin{aligned}
& c_{z}^{\mathrm{L} \rho}\left(\mathbf{r}, \mathbf{r}^{\prime} ; t, t^{\prime}\right)=i \frac{k_{\mathrm{B}} T}{\pi} \rho_{0} \eta \chi \int \frac{\mathrm{d} \omega^{\prime}}{\omega^{\prime}} e^{i \omega^{\prime}\left(t-t^{\prime}\right)} \\
& \times \int \mathrm{d} z^{\prime \prime} \int \frac{\mathrm{d}^{2} \mathbf{k}}{(2 \pi)^{2}} e^{i \mathbf{k} \cdot\left(\mathbf{s}-\mathbf{s}^{\prime}\right)} \nabla_{z}^{\prime \prime} \widetilde{G}^{\mathrm{L}}\left(z, z^{\prime \prime} ; \mathbf{k} ;-\omega^{\prime}\right) \\
& \times\left(\mathbf{k}^{2}+\nabla_{z}^{\prime} \nabla_{z}^{\prime \prime}\right) \widetilde{G}^{\mathrm{L}}\left(z^{\prime}, z^{\prime \prime} ;-\mathbf{k} ; \omega^{\prime}\right) .
\end{aligned}
$$

Now, following the line of reasoning of the previous section, the double integrals over $(x, y)$ and $\left(x^{\prime}, y^{\prime}\right)$ in the full contribution to the force correlator generate a Dirac delta function over the transverse wavenumbers, $(2 \pi)^{2} A \delta(\mathbf{k})$. Therefore, we can write this contribution in terms of the Green functions as

$$
\begin{aligned}
& \iint_{A} \mathrm{~d} A_{z} \mathrm{~d} A_{z}^{\prime} \nabla_{z} c_{z}^{\mathrm{L} \rho}\left(\mathbf{r}, \mathbf{r}^{\prime} ; t, t^{\prime}\right)=-\frac{k_{\mathrm{B}} T}{\pi} \rho_{0} \eta \chi A \\
& \times \int \frac{\mathrm{d} \omega^{\prime}}{\omega^{\prime}} \sin \left[\omega^{\prime}\left(t-t^{\prime}\right)\right] \int \mathrm{d} z^{\prime \prime} \nabla_{z} \nabla_{z}^{\prime \prime} \widetilde{G}^{\mathrm{L}}\left(z, z^{\prime \prime} ; \mathbf{0} ;-\omega^{\prime}\right) \\
& \quad \times \nabla_{z}^{\prime} \nabla_{z}^{\prime \prime} \widetilde{G}^{\mathrm{L}}\left(z^{\prime}, z^{\prime \prime} ; \mathbf{0} ; \omega^{\prime}\right) .
\end{aligned}
$$

Since this expression is odd with respect to changing $(z, t)$ to $\left(z^{\prime}, t^{\prime}\right)$ and vice versa, it follows that the contributions to the force correlator from the correlation functions between the density and velocity fields (third and fourth terms) in Eq. (A4) vanish, i.e.,

$$
\iint_{A} \mathrm{~d} A_{z} \mathrm{~d} A_{z}^{\prime}\left[\nabla_{z} c_{z}^{\mathrm{L} \rho}\left(\mathbf{r}, \mathbf{r}^{\prime} ; t, t^{\prime}\right)+\nabla_{z}^{\prime} c_{z}^{\mathrm{L} \rho}\left(\mathbf{r}^{\prime}, \mathbf{r} ; t^{\prime}, t\right)\right]=0 .
$$

\section{Appendix E: Derivation of the frequency integrals}

In this Appendix, we derive Eqs. (72) and (73) as well as Eqs. (67) and (68).

\section{Same-plate force correlator, Eq. 772}

We start with the first contribution to the force correlation function, which is given in Eq. (63):

$$
\begin{aligned}
& \mathcal{P}_{1}\left(z, z^{\prime} ; t, t^{\prime}\right)=\frac{k_{\mathrm{B}} T}{\pi} \eta^{3} \chi^{3} A \int \mathrm{d} \omega^{\prime} \cos \left[\omega^{\prime}\left(t-t^{\prime}\right)\right] \\
& \times \int \mathrm{d} z^{\prime \prime} \nabla_{z} \nabla_{z}^{\prime \prime} \widetilde{G}^{\mathrm{L}}\left(z, z^{\prime \prime} ; \mathbf{0} ;-\omega^{\prime}\right) \nabla_{z}^{\prime} \nabla_{z}^{\prime \prime} \widetilde{G}^{\mathrm{L}}\left(z^{\prime}, z^{\prime \prime} ; \mathbf{0} ; \omega^{\prime}\right) .
\end{aligned}
$$

The explicit expressions for the derivatives appearing on the right-hand side of the above equation are

$$
\begin{aligned}
& \nabla_{z} \nabla_{z}^{\prime \prime} \widetilde{G}^{\mathrm{L}}\left(z, z^{\prime \prime} ; \mathbf{0} ;-\omega\right)=\frac{-\omega}{2\left[i \rho_{0} c_{0}^{2}-(4 \eta / 3+\zeta) \omega\right]} \quad(\mathrm{E} 2) \\
& \times\left[-2 \delta\left(z-z^{\prime \prime}\right)+\lambda^{*} e^{-\lambda^{*}\left|z-z^{\prime \prime}\right|}+\lambda^{*} \operatorname{csch}\left(\lambda^{*} L\right)\right. \\
& \left.\times\left(\cosh \left(\lambda^{*}\left(L-z^{\prime \prime}\right)\right) e^{-\lambda^{*} z}+\cosh \left(\lambda^{*} z^{\prime \prime}\right) e^{\lambda^{*}(z-L)}\right)\right], \\
& \nabla_{z}^{\prime} \nabla_{z}^{\prime \prime} \widetilde{G}^{\mathrm{L}}\left(z^{\prime}, z^{\prime \prime} ; \mathbf{0} ; \omega\right)=\frac{\omega}{2\left[i \rho_{0} c_{0}^{2}+(4 \eta / 3+\zeta) \omega\right]} \quad(\mathrm{E} 3) \\
& \times\left[-2 \delta\left(z^{\prime}-z^{\prime \prime}\right)+\lambda e^{-\lambda\left|z^{\prime}-z^{\prime \prime}\right|}+\lambda \operatorname{csch}(\lambda L)\right. \\
& \left.\quad \times\left(\cosh \left(\lambda\left(L-z^{\prime \prime}\right)\right) e^{-\lambda z^{\prime}}+\cosh \left(\lambda z^{\prime \prime}\right) e^{\lambda\left(z^{\prime}-L\right)}\right)\right],
\end{aligned}
$$

where we have used the fact that $m=\lambda$ when $\mathbf{k}^{2}=0$ and we note that $\lambda^{*}=\lambda(-\omega)$.

Now, in principle, we could multiply together the results and integrate over $z^{\prime \prime}$. It is simpler, however, to look ahead a little. We know that, for the force variance at a single plate, we will ultimately evaluate this correlation function at $z=z^{\prime}=0$, so then these derivatives become

$$
\begin{aligned}
& \nabla_{z} \nabla_{z}^{\prime \prime} \widetilde{G}^{\mathrm{L}}\left(0, z^{\prime \prime} ; \mathbf{0} ;-\omega\right)=\frac{-\omega}{2\left[i \rho_{0} c_{0}^{2}-(4 \eta / 3+\zeta) \omega\right]} \\
& \times\left[-2 \delta\left(0-z^{\prime \prime}\right)+\lambda^{*} e^{-\lambda^{*} z^{\prime \prime}}+\lambda^{*} \operatorname{csch}\left(\lambda^{*} L\right)\right. \\
& \left.\quad \times\left(\cosh \left(\lambda^{*}\left(L-z^{\prime \prime}\right)\right)+\cosh \left(\lambda^{*} z^{\prime \prime}\right) e^{-\lambda^{*} L}\right)\right], \\
& \nabla_{z}^{\prime} \nabla_{z}^{\prime \prime} \widetilde{G}^{\mathrm{L}}\left(0, z^{\prime \prime} ; \mathbf{0} ; \omega\right)=\frac{\omega}{2\left[i \rho_{0} c_{0}^{2}+(4 \eta / 3+\zeta) \omega\right]} \\
& \times\left[-2 \delta\left(0-z^{\prime \prime}\right)+\lambda e^{-\lambda z^{\prime \prime}}+\lambda \operatorname{csch}(\lambda L)\right. \\
& \left.\quad \times\left(\cosh \left(\lambda\left(L-z^{\prime \prime}\right)\right)+\cosh \left(\lambda z^{\prime \prime}\right) e^{-\lambda L}\right)\right] .
\end{aligned}
$$

Here, we have simplified the expressions using $\left|-z^{\prime \prime}\right|=z^{\prime \prime}$ for $z^{\prime \prime}$ in the range $[0, L]$. 
The key simplification now is to notice that we can collect together many of the exponential terms, which simplify to give

$$
\begin{aligned}
& \nabla_{z} \nabla_{z}^{\prime \prime} \widetilde{G}^{\mathrm{L}}\left(0, z^{\prime \prime} ; \mathbf{0} ;-\omega\right)=\frac{-\omega}{\left[i \rho_{0} c_{0}^{2}-(4 \eta / 3+\zeta) \omega\right]} \\
& \quad \times\left[\lambda^{*} \cosh \left[\lambda^{*}\left(L-z^{\prime \prime}\right)\right] \operatorname{csch}\left[\lambda^{*} L\right]-\delta\left(0-z^{\prime \prime}\right)\right] \\
& \nabla_{z}^{\prime} \nabla_{z}^{\prime \prime} \widetilde{G}^{\mathrm{L}}\left(0, z^{\prime \prime} ; \mathbf{0} ; \omega\right)=\frac{\omega}{\left[i \rho_{0} c_{0}^{2}+(4 \eta / 3+\zeta) \omega\right]} \\
& \quad \times\left[\lambda \cosh \left[\lambda\left(L-z^{\prime \prime}\right)\right] \operatorname{csch}[\lambda L]-\delta\left(0-z^{\prime \prime}\right)\right]
\end{aligned}
$$

Let us now use this result in our full expression, giving

$$
\begin{gathered}
\mathcal{P}_{1}\left(0,0 ; t, t^{\prime}\right)=\frac{k_{\mathrm{B}} T}{\pi} \eta^{3} \chi^{3} A \int \frac{\mathrm{d} \omega \omega^{2} \cos \left[\omega\left(t-t^{\prime}\right)\right]}{\rho_{0}^{2} c_{0}^{4}+(4 \eta / 3+\zeta)^{2} \omega^{2}} \\
\times \int_{0}^{L} \mathrm{~d} z^{\prime \prime}\left[\lambda^{*} \cosh \left[\lambda^{*}\left(L-z^{\prime \prime}\right)\right] \operatorname{csch}\left[\lambda^{*} L\right]-\delta\left(0-z^{\prime \prime}\right)\right] \\
\times\left[\lambda \cosh \left[\lambda\left(L-z^{\prime \prime}\right)\right] \operatorname{csch}[\lambda L]-\delta\left(0-z^{\prime \prime}\right)\right] \\
=\frac{k_{\mathrm{B}} T}{\pi} \eta^{3} \chi^{3} A \int \frac{\mathrm{d} \omega \omega^{2} \cos \left[\omega\left(t-t^{\prime}\right)\right]}{\rho_{0}^{2} c_{0}^{4}+(4 \eta / 3+\zeta)^{2} \omega^{2}} \\
\times\left[\frac{|\lambda|^{2}\left(\lambda_{\mathrm{R}} \sin \left[2 \lambda_{\mathrm{I}} L\right]+\lambda_{\mathrm{I}} \sinh \left[2 \lambda_{\mathrm{R}} L\right]\right)}{2 \lambda_{\mathrm{I}} \lambda_{\mathrm{R}}\left(\cosh \left[2 \lambda_{\mathrm{R}} L\right]-\cos \left[2 \lambda_{\mathrm{I}} L\right]\right)}\right. \\
\left.\quad-\frac{2\left(\lambda_{\mathrm{I}} \sin \left[2 \lambda_{\mathrm{I}} L\right]+\lambda_{\mathrm{R}} \sinh \left[2 \lambda_{\mathrm{R}} L\right]\right)}{\left(\cosh \left[2 \lambda_{\mathrm{R}} L\right]-\cos \left[2 \lambda_{\mathrm{I}} L\right]\right)}+\delta(0)\right] . \quad(\mathrm{E} 8)
\end{gathered}
$$

We note that, in the $L \rightarrow \infty$ limit, this result reduces to the expression for the semi-infinite fluid, Eq. (F3), an important cross-check of our results. We now express our result in terms of the dimensionless parameters $\ell_{+}, \ell_{-}$, $\chi, \gamma$, and $\tau=\left(t-t^{\prime}\right) / t_{0}$, the dimensionless variable $u$ and the function $f_{m}(u)$ (see Sec. IVB):

$$
\begin{aligned}
\mathcal{P}_{1}\left(0,0 ; t, t^{\prime}\right)=\frac{k_{\mathrm{B}} T}{\pi} \rho_{0} c_{0}^{2} \chi^{3} \frac{A}{L} 2 \int_{0}^{u_{\infty}} \mathrm{d} u f_{0}(u) \cos [u \tau] \\
\times\left\{\frac { 1 } { \operatorname { c o s h } [ 2 \ell _ { + } ] - \operatorname { c o s } [ 2 \ell _ { - } ] } \left[\left(\frac{\ell^{2}}{2 \ell_{-}}-2 \ell_{-}\right) \sin \left[2 \ell_{-}\right]\right.\right. \\
\left.\left.\quad+\left(\frac{\ell^{2}}{2 \ell_{+}}-2 \ell_{+}\right) \sinh \left[2 \ell_{+}\right]\right]+L \delta(0)\right\} .
\end{aligned}
$$

Here, $\ell^{2}=\ell_{+}^{2}+\ell_{-}^{2}$ and we have used the fact that the integrand is symmetric in the frequency to rewrite the region of integration over the positive real axis only, up to the dimensionless cutoff, $u_{\infty}=\delta^{2} / a^{2}$. We now define

$$
\begin{aligned}
& \mathcal{W}_{0}(0,0 ; \tau)=2 \int_{0}^{u_{\infty}} \mathrm{d} u f_{0}(u) \cos [u \tau] \\
& \times \frac{1}{\cosh \left[2 \ell_{+}\right]-\cos \left[2 \ell_{-}\right]}\left[\left(\frac{\ell^{2}}{2 \ell_{-}}-2 \ell_{-}\right) \sin \left[2 \ell_{-}\right]\right. \\
& \left.\quad+\left(\frac{\ell^{2}}{2 \ell_{+}}-2 \ell_{+}\right) \sinh \left[2 \ell_{+}\right]\right]
\end{aligned}
$$

and

$$
\mathcal{V}_{0}(0,0 ; \tau)=2 \int_{0}^{u_{\infty}} \mathrm{d} u f_{0}(u) \cos [u \tau]
$$

and thus we have

$$
\begin{aligned}
\mathcal{P}_{1}(0,0 ; & \left.t, t^{\prime}\right)=\frac{k_{\mathrm{B}} T}{\pi} \rho_{0} c_{0}^{2} \chi^{3} \frac{A}{L} \\
\times & {\left[\mathcal{W}_{0}(0,0 ; \tau)+L \mathcal{V}_{0}(0,0 ; \tau) \delta(0)\right] . }
\end{aligned}
$$

Now, we turn to the second contribution, $\mathcal{P}_{2}\left(0,0 ; t, t^{\prime}\right)$. The derivatives with respect to $x$ and $y$ will ultimately bring down factors of $k_{x}$ and $k_{y}$. When we integrate over the spatial directions, the Dirac delta functions in wavenumber will remove these terms. The result is then directly related to the equation above, except that there is an extra denominator of $\omega^{2}$. We thus find

$$
\begin{aligned}
\mathcal{P}_{2}\left(0,0 ; t, t^{\prime}\right)=\frac{k_{\mathrm{B}} T}{\pi} \rho_{0} c_{0}^{2} \chi \frac{A}{L} 2 \int_{0}^{u_{\infty}} \mathrm{d} u f_{2}(u) \cos [u \tau] \\
\times\left\{\frac { 1 } { \operatorname { c o s h } [ 2 \ell _ { + } ] - \operatorname { c o s } [ 2 \ell _ { - } ] } \left[\left(\frac{\ell^{2}}{2 \ell_{-}}-2 \ell_{-}\right) \sin \left[2 \ell_{-}\right]\right.\right. \\
\left.\left.\quad+\left(\frac{|\lambda|^{2}}{2 \ell_{+}}-2 \ell_{+}\right) \sinh \left[2 \ell_{+}\right]\right]+L \delta(0)\right\},
\end{aligned}
$$

or, in terms of the frequency integrals $\mathcal{W}_{2}$, Eq. (67), and $\mathcal{V}_{2}$, Eq. (69), we have

$$
\begin{aligned}
\mathcal{P}_{2}\left(0,0 ; t, t^{\prime}\right) & =\frac{k_{\mathrm{B}} T}{\pi} \rho_{0} c_{0}^{2} \chi \frac{A}{L} \\
& \times\left[\mathcal{W}_{2}(0,0 ; \tau)+L \mathcal{V}_{2}(0,0 ; \tau) \delta(0)\right]
\end{aligned}
$$

This, too, reduces to the expression for the semi-infinite fluid, Eq. (F4), in the $L \rightarrow \infty$ limit.

Finally, putting together Eqs. (E12) and (E14) and using Eq. (71), we obtain Eq. (72).

\section{Cross-plate force correlator, Eq. (73)}

Here we derive Eq. (73), our final integral expression for the cross-plate force correlator. We start from Eqs. (E1), (E3) and (E4) again, but now we need to evaluate one of the derivatives at $z^{\prime}=L$,

$$
\begin{aligned}
\nabla_{z} \nabla_{z}^{\prime \prime} \widetilde{G}^{\mathrm{L}}\left(0, z^{\prime \prime} ; \mathbf{0} ;-\omega\right)=\frac{-\omega}{2\left[i \rho_{0} c_{0}^{2}-(4 \eta / 3+\zeta) \omega\right]} \\
\times\left[-2 \delta\left(0-z^{\prime \prime}\right)+\lambda^{*} e^{-\lambda^{*} z^{\prime \prime}}+\lambda^{*} \operatorname{csch}\left(\lambda^{*} L\right)\right. \\
\left.\quad \times\left(\cosh \left(\lambda^{*}\left(L-z^{\prime \prime}\right)\right)+\cosh \left(\lambda^{*} z^{\prime \prime}\right) e^{-\lambda^{*} L}\right)\right] \\
\nabla_{z}^{\prime} \nabla_{z}^{\prime \prime} \widetilde{G}^{\mathrm{L}}\left(L, z^{\prime \prime} ; \mathbf{0} ; \omega\right)=\frac{\omega}{2\left[i \rho_{0} c_{0}^{2}+(4 \eta / 3+\zeta) \omega\right]} \\
\times\left[-2 \delta\left(L-z^{\prime \prime}\right)+\lambda e^{-\lambda\left(L-z^{\prime \prime}\right)}+\lambda \operatorname{csch}(\lambda L)\right. \\
\left.\quad \times\left(\cosh \left(\lambda\left(L-z^{\prime \prime}\right)\right) e^{-\lambda L}+\cosh \left(\lambda z^{\prime \prime}\right)\right)\right]
\end{aligned}
$$


Once again we can simplify matters by writing

$$
\begin{aligned}
& \nabla_{z} \nabla_{z}^{\prime \prime} \widetilde{G}^{\mathrm{L}}\left(0, z^{\prime \prime} ; \mathbf{0} ;-\omega\right)=\frac{-\omega}{i \rho_{0} c_{0}^{2}-(4 \eta / 3+\zeta) \omega} \\
& \times\left[\lambda^{*} \cosh \left[\lambda^{*}\left(L-z^{\prime \prime}\right)\right] \operatorname{csch}\left[\lambda^{*} L\right]-\delta\left(0-z^{\prime \prime}\right)\right],
\end{aligned}
$$

but, in this case, Eq. (E16) becomes

$$
\begin{aligned}
\nabla_{z}^{\prime} \nabla_{z}^{\prime \prime} \widetilde{G}^{\mathrm{L}}\left(L, z^{\prime \prime} ; \mathbf{0} ; \omega\right)=\frac{\omega}{i \rho_{0} c_{0}^{2}+(4 \eta / 3+\zeta) \omega} \\
\times\left[\lambda \cosh \left[\lambda z^{\prime \prime}\right] \operatorname{csch}[\lambda L]-\delta\left(L-z^{\prime \prime}\right)\right] .
\end{aligned}
$$

Therefore, the first contribution to the force correlator between the two plates is

$$
\begin{gathered}
\mathcal{P}_{1}\left(0, L ; t, t^{\prime}\right)=\frac{k_{\mathrm{B}} T}{\pi} \eta^{3} \chi^{3} A \int \frac{\mathrm{d} \omega \omega^{2} \cos \left[\omega\left(t-t^{\prime}\right)\right]}{\rho_{0}^{2} c_{0}^{4}+(4 \eta / 3+\zeta)^{2} \omega^{2}} \\
\times \int_{0}^{L} \mathrm{~d} z^{\prime \prime}\left[\lambda^{*} \cosh \left[\lambda^{*}\left(L-z^{\prime \prime}\right)\right] \operatorname{csch}\left[\lambda^{*} L\right]-\delta\left(0-z^{\prime \prime}\right)\right] \\
\times\left[\lambda \cosh \left[\lambda z^{\prime \prime}\right] \operatorname{csch}[\lambda L]-\delta\left(L-z^{\prime \prime}\right)\right] \\
=\frac{k_{\mathrm{B}} T}{\pi} \eta^{3} \chi^{3} A \int \frac{\mathrm{d} \omega \omega^{2} \cos \left[\omega\left(t-t^{\prime}\right)\right]}{\rho_{0}^{2} c_{0}^{4}+(4 \eta / 3+\zeta)^{2} \omega^{2}} \\
\times\left[\frac{|\lambda|^{2}\left(\lambda_{\mathrm{R}} \cosh \left[\lambda_{\mathrm{R}} L\right] \sin \left[\lambda_{\mathrm{I}} L\right]+\lambda_{\mathrm{I}} \cos \left[\lambda_{\mathrm{I}} L\right] \sinh \left[\lambda_{\mathrm{R}} L\right]\right)}{\lambda_{\mathrm{I}} \lambda_{\mathrm{R}}\left(\cosh \left[2 \lambda_{\mathrm{R}} L\right]-\cos \left[2 \lambda_{\mathrm{I}} L\right]\right)}\right. \\
\left.-\frac{4\left(\lambda_{\mathrm{I}} \cosh \left[\lambda_{\mathrm{R}} L\right] \sin \left[\lambda_{\mathrm{I}} L\right]+\lambda_{\mathrm{R}} \cos \left[\lambda_{\mathrm{I}} L\right] \sinh \left[\lambda_{\mathrm{R}} L\right]\right)}{\cosh \left[2 \lambda_{\mathrm{R}} L\right]-\cos \left[2 \lambda_{\mathrm{I}} L\right]}\right] .
\end{gathered}
$$

We now express our result in terms of the dimensionless parameters as before, giving

$$
\begin{gathered}
\mathcal{P}_{1}\left(0, L ; t, t^{\prime}\right)=\frac{k_{\mathrm{B}} T}{\pi} \rho_{0} c_{0}^{2} \chi^{3} \frac{A}{L} 2 \int_{0}^{u_{\infty}} \mathrm{d} u f_{0}(u) \cos [u \tau] \\
\times \frac{1}{\cosh \left[2 \ell_{+}\right]-\cos \left[2 \ell_{-}\right]}\left[\left(\frac{\ell^{2}}{\ell_{-}}-4 \ell_{-}\right) \cosh \left[\ell_{+}\right] \sin \left[\ell_{-}\right]\right. \\
\left.\quad+\left(\frac{\ell^{2}}{\ell_{+}}-4 \ell_{+}\right) \cos \left[\ell_{-}\right] \sinh \left[\ell_{+}\right]\right] .
\end{gathered}
$$

As in the case of the same-plate force correlator, the other contribution, $\mathcal{P}_{2}\left(0, L ; t, t^{\prime}\right)$, is very simply related to $\mathcal{P}_{1}\left(0, L ; t, t^{\prime}\right)$. We can write the result immediately as

$$
\begin{gathered}
\mathcal{P}_{2}\left(0, L ; t, t^{\prime}\right)=\frac{k_{\mathrm{B}} T}{\pi} \rho_{0} c_{0}^{2} \chi \frac{A}{L} 2 \int_{0}^{u_{\infty}} \mathrm{d} u f_{2}(u) \cos [u \tau] \\
\times \frac{1}{\cosh \left[2 \ell_{+}\right]-\cos \left[2 \ell_{-}\right]}\left[\left(\frac{\ell^{2}}{\ell_{-}}-4 \ell_{-}\right) \cosh \left[\ell_{+}\right] \sin \left[\ell_{-}\right]\right. \\
\left.\quad+\left(\frac{\ell^{2}}{\ell_{+}}-4 \ell_{+}\right) \cos \left[\ell_{-}\right] \sinh \left[\ell_{+}\right]\right] .
\end{gathered}
$$

Now, putting together Eqs. (E20) and (E21) and defining the frequency integral

$$
\begin{gathered}
\mathcal{W}_{m}(0, L ; \tau)=2 \int_{0}^{u_{\infty}} \mathrm{d} u f_{m}(u) \cos [u \tau] \\
\times \frac{1}{\cosh \left[2 \ell_{+}\right]-\cos \left[2 \ell_{-}\right]}\left[\left(\frac{\ell^{2}}{\ell_{-}}-4 \ell_{-}\right) \cosh \left[\ell_{+}\right] \sin \left[\ell_{-}\right]\right. \\
\left.\quad+\left(\frac{\ell^{2}}{\ell_{+}}-4 \ell_{+}\right) \cos \left[\ell_{-}\right] \sinh \left[\ell_{+}\right]\right], \quad \quad(\mathrm{E} 22)
\end{gathered}
$$

we obtain Eq. (73).

In the large plate-separation limit, $L \rightarrow \infty$, these correlators vanish, in accordance with the results of Appendix F1.

\section{Appendix F: Time correlators for simple geometries}

In this Appendix, we derive the time-dependent correlators for two simple geometries: A semi-infinite fluid with a single hard-wall boundary and an infinite fluid. The semi-infinite fluid is the limiting case for the twowall geometry in the limit of infinite plate separation and we have confirmed, both analytically and numerically, that our results for the two-wall geometry reduce to the semi-infinite fluid results.

\section{Semi-infinite fluid}

The Green function solution of Eq. (40) for a semiinfinite fluid, with an infinite hard-wall boundary at $z=$ 0 , is

$$
\widetilde{G}^{\mathrm{L}}\left(z, z^{\prime \prime} ; \mathbf{k} ; \omega\right)=\frac{i \lambda^{2}}{2 m \omega \rho_{0}}\left[e^{-m\left(z+z^{\prime \prime}\right)}-e^{-m\left|z-z^{\prime \prime}\right|}\right],
$$

where now $z^{\prime \prime}>0$. Once again, we substitute this result into Eqs. (63) and (64). The derivative we require this time is

$$
\begin{aligned}
\nabla_{z} \nabla_{z}^{\prime \prime} \widetilde{G}^{\mathrm{L}}\left(z, z^{\prime \prime} ; \mathbf{k} ; \omega\right)=\frac{i \lambda^{2}}{2 \omega \rho_{0}} & {\left[m e^{-m\left(z+z^{\prime \prime}\right)}+m e^{-m\left|z-z^{\prime \prime}\right|}\right.} \\
& \left.-2 \delta\left(z-z^{\prime \prime}\right)\right] .
\end{aligned}
$$

Carrying out the spatial integrals over $z^{\prime \prime}$, from zero to infinity, we obtain

$$
\begin{aligned}
\mathcal{P}_{1}\left(0,0 ; t, t^{\prime}\right)=\frac{k_{\mathrm{B}} T}{\pi} \frac{\eta^{3} \chi^{3} A}{\rho_{0}^{2}} \int \frac{\mathrm{d} \omega^{\prime}}{\omega^{\prime 2}}|\lambda|^{4} \cos \left[\omega^{\prime}\left(t-t^{\prime}\right)\right] \\
\times\left[\frac{|\lambda|^{2}}{2 \lambda_{\mathrm{R}}}-2 \lambda_{\mathrm{R}}+\delta(0)\right], \\
\mathcal{P}_{2}\left(0,0 ; t, t^{\prime}\right)=\frac{k_{\mathrm{B}} T}{\pi} \eta \chi c_{0}^{4} A \int \frac{\mathrm{d} \omega^{\prime}}{\omega^{\prime 4}}|\lambda|^{4} \cos \left[\omega^{\prime}\left(t-t^{\prime}\right)\right] \\
\times\left[\frac{|\lambda|^{2}}{2 \lambda_{\mathrm{R}}}-2 \lambda_{\mathrm{R}}+\delta(0)\right] .
\end{aligned}
$$


We can carry out these frequency integrals for the equal time case, with $t=t^{\prime}$, by first transforming to the dimensionless variables, $x=\eta \chi \omega /\left(\rho_{0} c_{0}^{2}\right)$ and $\tau_{x}=$ $\left(t-t^{\prime}\right) \rho_{0} c_{0}^{2} /(\eta \chi)$, and then defining $\tan \theta=x$. We obtain

$$
\begin{gathered}
\mathcal{P}_{1}(0,0)=\frac{k_{\mathrm{B}} T}{\pi} 2 \rho_{0} c_{0}^{2} A\left\{\delta(0) \int_{0}^{x_{\infty}} \frac{\mathrm{d} x x^{2}}{x^{2}+1}\right. \\
\left.+\frac{\rho_{0} c_{0}}{\sqrt{2} \eta \chi} \int_{0}^{\theta \infty} \mathrm{d} \theta \tan ^{3} \theta \frac{(2 \cos \theta-1)}{\sqrt{\sec \theta-1}}\right\} \\
=\frac{k_{\mathrm{B}} T}{\pi} 2 \rho_{0} c_{0}^{2} A\left\{\delta(0)\left(x_{\infty}-\arctan x_{\infty}\right)\right. \\
\left.+\frac{8 \sqrt{2} \rho_{0} c_{0}}{3 \eta \chi} \frac{z_{\infty}\left(3-z_{\infty}\right)}{\sqrt{z_{\infty}-1}} \sin ^{4}\left(\frac{1}{2} \arctan \left(z_{\infty}^{2}-1\right)\right)\right\},
\end{gathered}
$$

and

$$
\begin{aligned}
& \mathcal{P}_{2}(0,0)=\frac{k_{\mathrm{B}} T}{\pi} 2 \rho_{0} c_{0}^{2} A\left\{\delta(0) \int_{0}^{x_{\infty}} \frac{\mathrm{d} x}{x^{2}+1}\right. \\
& \left.+\frac{\rho_{0} c_{0}}{\sqrt{2} \eta \chi} \int_{0}^{\theta} \mathrm{d} \theta \tan \theta \frac{(2 \cos \theta-1)}{\sqrt{\sec \theta-1}}\right\} \\
& =\frac{k_{\mathrm{B}} T}{\pi} 2 \rho_{0} c_{0}^{2} A\left\{\delta(0) \arctan x_{\infty}+\frac{2 \rho_{0} c_{0}}{\eta \chi} \frac{\sqrt{z_{\infty}-1}}{z_{\infty}}\right\} .
\end{aligned}
$$

Here, $\theta_{\infty}=\arctan x_{\infty}$ and $z_{\infty}=\sqrt{1+x_{\infty}^{2}}$ are both functions of the dimensionless cutoff $x_{\infty}=\eta^{2} \chi /\left(a^{2} \rho_{0}^{2} c_{0}^{2}\right)$.

The equal-time correlator for a semi-infinite fluid is then given by

$$
\begin{gathered}
\mathcal{C}(0,0)=\frac{k_{\mathrm{B}} T}{\pi} 2 \rho_{0} c_{0}^{2} A\left\{x_{\infty} \cdot \delta(0)+\frac{\rho_{0} c_{0}}{\eta \chi}\left[\frac{2 \sqrt{z_{\infty}-1}}{z_{\infty}}\right.\right. \\
\left.\left.+\frac{8 \sqrt{2}}{3} \frac{z_{\infty}\left(3-z_{\infty}\right)}{\sqrt{z_{\infty}-1}} \sin ^{4}\left(\frac{1}{2} \arctan \left(z_{\infty}^{2}-1\right)\right)\right]\right\},
\end{gathered}
$$

which is Eq. (78).

The corresponding time-dependent, cross-plate force correlator vanishes for the semi-infinite fluid geometry, dropping to zero as $1 / L$.

\section{Infinite fluid}

The Green function solution of Eq. (40) for an infinite fluid, i.e., vanishing Green function at $z \rightarrow \pm \infty$, is

$$
\widetilde{G}^{\mathrm{L}}\left(z, z^{\prime \prime} ; \mathbf{k} ; \omega\right)=-\frac{i \lambda^{2}}{2 m \omega \rho_{0}} e^{-m\left|z-z^{\prime \prime}\right|},
$$

where $m^{2}=\mathbf{k}^{2}+\lambda^{2}$ and $\lambda$ is the longitudinal decay constant defined in Eq. (41). We will substitute this result into Eqs. (63) and (64). The derivative we require is

$$
\begin{aligned}
\nabla_{z} \nabla_{z}^{\prime \prime} \widetilde{G}^{\mathrm{L}}\left(z, z^{\prime \prime} ; \mathbf{k} ; \omega\right)=\frac{i \lambda^{2}}{2 \omega \rho_{0}}\left[m e^{-m\left|z-z^{\prime \prime}\right|}\right. & \\
& \left.-2 \delta\left(z-z^{\prime \prime}\right)\right] .
\end{aligned}
$$

For an infinite fluid, the spatial integral runs from negative infinity to positive infinity and we assume that we determine the time correlator at $z=z^{\prime}=0$. Carrying out the spatial integral, we obtain

$$
\begin{aligned}
& \mathcal{P}_{1}\left(0,0 ; t, t^{\prime}\right)=\frac{k_{\mathrm{B}} T}{\pi} \frac{\eta^{3} \chi^{3} A}{\rho_{0}^{2}} \int \frac{\mathrm{d} \omega^{\prime}}{\omega^{\prime 2}}|\lambda|^{4} \cos \left[\omega^{\prime}\left(t-t^{\prime}\right)\right] \\
& \times\left[\frac{|\lambda|^{2}}{4 \lambda_{\mathrm{R}}}-\lambda_{\mathrm{R}}+\delta(0)\right], \quad(\mathrm{F} 10) \\
& \mathcal{P}_{2}\left(0,0 ; t, t^{\prime}\right)=\frac{k_{\mathrm{B}} T}{\pi} \eta \chi c_{0}^{4} A \int \frac{\mathrm{d} \omega^{\prime}}{\omega^{\prime 4}}|\lambda|^{4} \cos \left[\omega^{\prime}\left(t-t^{\prime}\right)\right] \\
& \times\left[\frac{|\lambda|^{2}}{4 \lambda_{\mathrm{R}}}-\lambda_{\mathrm{R}}+\delta(0)\right]
\end{aligned}
$$

By comparing these results to Eqs. (F3) and (F4), we immediately see that this result is simply half that of the semi-infinite fluid. Therefore, we have

$$
\left.\mathcal{C}\left(0,0 ; t, t^{\prime}\right)\right|_{\text {infinite }}=\left.\frac{1}{2} \mathcal{C}\left(0,0 ; t, t^{\prime}\right)\right|_{\text {semi-infinite }}
$$

[1] H.B.G. Casimir, Proc. K. Ned. Akad. Wet. 51, 793 (1948).

[2] V.M. Mostepanenko and N.N. Trunov, The Casimir Effect and Its Applications (Clarendon, Oxford, 1997).

[3] M. Kardar and R. Golestanian, Rev. Mod. Phys. 71, 1233 (1999).

[4] M. Bordag, U. Mohideen and V.M. Mostepanenko, Phys. Rep. 353, 2 (2001).

[5] V.A. Parsegian, Van der Waals Forces: A Handbook for Biologists, Chemists, Engineers, and Physicists (Cambridge University Press, 2005).

[6] M. Bordag, G.L. Klimchitskaya, U. Mohideen and V.M. Mostepanenko, Advances in the Casimir Effect (Oxford University Press, New York, 2009).
[7] D.A.R. Dalvit, P.W. Milonni, D. Roberts, F.S.S. Rosa, Casimir Physics, Lecture Notes in Physics, Vol. 834, (Springer-Verlag, Berlin, 2011).

[8] M. Krech, The Casimir Effect in Critical Systems (World Scientific, Singapore, 1994).

[9] R. French et al., Rev. Mod. Phys. 82, 1887 (2010).

[10] M.E. Fisher and P.G. de Gennes, C. R. Acad. Sci. Paris B 287, 207 (1978).

[11] M. Krech, J. Phys. Condens. Matter 11, 391 (1999).

[12] M. Krech, Phys. Rev. E 56, 1642 (1997).

[13] C. Hertlein, L. Helden, A. Gambassi, S. Dietrich and C. Bechinger, Nature 451, 172 (2008).

[14] M. Fukuto, Y.F. Yano, and P.S. Pershan, Phys. Rev. Lett. 94, 135702 (2005). 
[15] E. Schaeffer and U. Steiner, Eur. Phys. J. E 8, 347 (2002).

[16] M.D. Morariu, E. Schaeffer and U. Steiner, Phys. Rev. Lett. 92, 156102 (2004).

[17] M.D. Morariu, E. Schaeffer and U. Steiner, Eur. Phys. J. E 12, 375 (2003).

[18] A. Ajdari, L. Peliti and J. Prost, Phys. Rev. Lett. 66, 1481 (1991).

[19] H. Li and M. Kardar, Phys. Rev. Lett. 67, 3275 (1991).

[20] M. Antezza, L.P. Pitaevskii, S. Stringari, and V.B. Svetovoy, Phys. Rev. A 77, 022901 (2008).

[21] M. Krüger, T. Emig and M. Kardar, Phys. Rev. Lett. 106 201404 (2011).

[22] D.S. Dean, V.A. Parsegian, and R. Podgornik, Phys. Rev. A 87, 032111 (2013).

[23] T.R. Kirkpatrick, J.M. Ortiz de Zárate, J.V. Sengers, Phys. Rev. Lett. 110, 235 (2013).

[24] T.R. Kirkpatrick, J.M. Ortiz de Zárate and J.V. Sengers, Phys. Rev. E 89, 022145 (2014).

[25] J.M. Ortiz de Zárate and J.V. Sengers, Hydrodynamic Fluctuations in Fluids and Fluid Mixtures (Elsevier, Amsterdam, 2006).

[26] E.M. Lifshitz, Sov. Phys. JETP 2, 73 (1956).

[27] S.M. Rytov, Theory of Electric Fluctuations and Thermal Radiation (AFCRC-TR Air Force Cambridge Research Center, Bedford, 1959)

[28] F.S.S. Rosa, D.A.R. Dalvit, and P.W. Milonni, Phys. Rev. A 81, 033812 (2010).

[29] A. Narayanaswamy and Yi Zheng, Phys. Rev. A 88, $012502(2013)$.

[30] D.S. Dean and A. Gopinathan, Phys. Rev. E 81, 041126 (2010).

[31] D.S. Dean and A. Gopinathan, J. Stat. Mech. L08001 (2009).

[32] L.D. Landau and E.M. Lifshitz, Statistical Physics, Part 2, 1st Ed. (Butterworth-Heinemann, Woburn, 1996).

[33] D. Forster, Hydrodynamic Fluctuations, Broken Symmetry, and Correlation Functions (W.A. Benjamin, Reading, 1975).

[34] H. Spohn, J. Phys. A: Math. Gen. 16, 4275 (1983).

[35] J. Tailleur, J. Kurchan, and V. Lecomte, J. Phys. A: Math. Theor. 41, 505001 (2008).

[36] J.S. Rowlinson, Cohesion: A Scientific History of In- termolecular Forces (Cambridge University Press, Cambridge, 2002).

[37] R.B. Jones, Physica A 105, 395 (1981).

[38] D.Y.C. Chan, L.R. White, Physica A 122, 505 (1983).

[39] N.G. van Kampen and J.J. Lodder, Constraints, Am. J. Phys. 52, 419-424 (1984).

[40] I.E. Dzyaloshinskii, E.M. Lifshitz, and L.P. Pitaevskii, Sov. Phys. Uspekhi 4, 153 (1961).

[41] B.I. Ivlev, J. Phys. Condens. Matter 14, 4829 (2002).

[42] D. Bartolo, A. Ajdari, J-B. Fournier, and R. Golestanian, Phys. Rev. Lett. 89, 230601 (2002).

[43] A. Naji, D.S. Dean, J. Sarabadani, R. Horgan, R. Podgornik, Phys. Rev. Lett. 104, 060601 (2010).

[44] D.S. Dean, A. Naji and R. Podgornik, Phys. Rev. E 83, 011102 (2011).

[45] A. Naji, J. Sarabadani, D.S. Dean and R. Podgornik, Eur. Phys. J. E 35, 24 (2012).

[46] We note the typographic error in Eq. (2.1) of Ref. [38], a missing occurrence of the field $\mathbf{v}$.

[47] Here we note a typographic error in Eq. (2.11) of 38], corresponding to a factor of $1 / \rho_{0}$ missing from Eq. (13) above.

[48] A. Erbas, R. Podgornik and R.R. Netz, Eur. Phys. J. E 32, 147 (2010).

[49] S. Kim and S. Karrila Microhydrodynamics, 1st Ed. (Dover, Mineola, 2005).

[50] J. Schwinger, L.L. Deraad Jr., K.A. Milton, W. Tsai and J. Norton, Classical Electrodynamics (Westview, Boulder, 1998).

[51] U. Raviv, P. Laurat and J. Klein, Nature 413, 5154 (2001).

[52] U. Raviv and J. Klein, Science 297, 1540 (2002).

[53] Y. Leng and P.T. Cummings, Phys. Rev. Lett. 94, 026101 (2005).

[54] C. De Bacco, F. Baldovin, E. Orlandini, and K. Sekimoto, Phys. Rev. Lett. 112, 180605 (2014).

[55] J. von Hansen, A. Mehlich, B. Pelz, M. Rief, and R.R. Netz, Rev. Sci. Inst. 83, 095116 (2012).

[56] S. Lise, A. Maritan and M.R. Swift, J. Phys. A: Math. Gen. 32, 5251 (1999). 